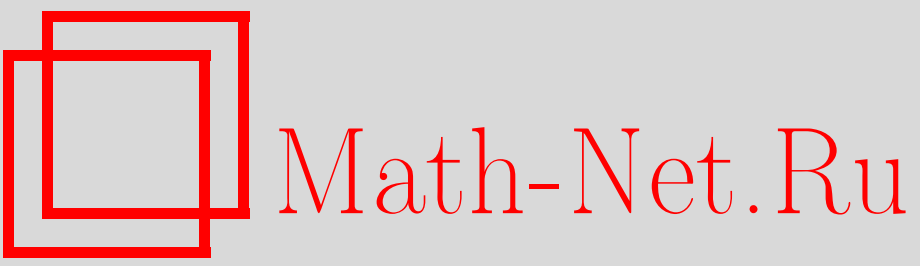

С. А. Богатый, В. М. Вылов, Вложения Робертса и обращение трансверсальной теоремы Тверберга, Матем. сб., 2005, том 196, номер 11, 33-52

DOI: https://doi.org/10.4213/sm1390

Использование Общероссийского математического портала Math-Net.Ru подразумевает, что вы прочитали и согласны с пользовательским соглашением

http://www.mathnet.ru/rus/agreement

Параметры загрузки:

IP: 54.162 .127 .20

26 апреля 2023 г., 15:57:26 


\author{
С.А. Богатый, В.М. Вылов
}

\title{
Вложения Робертса и обращение трансверсальной теоремы Тверберга
}

\begin{abstract}
Главньми результатами статьи являются два утверждения о существовании "экономичных вложений" в евклидово пространство. В первом результате (следствие 1.4) доказывается, что существует вложение, при котором образ пересекается с плоскостями большой размерности по множествам "контролируемой" размерности. Во втором результате (следствие 1.6) доказьвается существование отображений, при которых на каждую плоскость небольшой размерности попадает "контролируемое" число точек.

В качестве следствий наших результатов вьводятся известные теоремы Нёбелинга-Понтрягина, Робертса, Гуревича, Болтянского и Гудселла. Получен также бесконечномерный вариант о вложении в гильбертово пространство (теорема 1.8).

Библиографиия: 31 название.
\end{abstract}

\section{§ 1. Введение}

Все рассматриваемые в настоящей работе отображения предполагаются непрерывными, а все пространства предполагаются, по крайней мере, вполне регулярньми. Всюду ниже $\Pi^{d} \subset \mathbb{R}^{m}$ обозначает $d$-мерную, не обязательно координатную плоскость (просто $d$-плоскость) в $\mathbb{R}^{m}$. Если специально не оговорено противное, то функциональное пространство в работе всегда наделяется основной предельной топологией [1].

Нашей целью является доказательство нижеследующей теоремы 1.1 и получение некоторых следствий этой теоремы.

Теорема 1.1. Пусть $A_{i, j}, i=1,2, \ldots, q, j=1,2, \ldots, n_{i}+1,-$ такие точки в $\mathbb{R}^{m}$, что множсество их координат алгебраически независимо. Пусть $0 \leqslant$ $t \leqslant d \leqslant T \leqslant m u \Pi^{d}-d$-мерная плоскость в $\mathbb{R}^{m}$, параллельная некоторымм координатным плоскостям $\Pi^{t} \subset \Pi^{T} \subset \mathbb{R}^{m}$. Eсли $d-t+1 \leqslant q u n_{1}+n_{2}+$ $\cdots+n_{q}+1 \leqslant(m-d)(q-1)-(T-d)(d-t)$ или $q \leqslant d-t+1$ u $n_{1}+n_{2}+$ $\cdots+n_{q}+1 \leqslant(m-T)(q-1)$, то существует такой индекс $i \in\{1,2, \ldots, q\}$, что плоскость $\Pi^{d}$ не пересекает линейную оболочку $\Pi\left(M_{i}\right)$ множества $M_{i}=$ $\left\{A_{i, 1}, \ldots, A_{i, n_{i}+1}\right\}$.

Первая часть теоремы 1.1 , когда $t=0, T=m$ и $d-t+1 \leqslant q$, была высказана в качестве гипотезы в работах [2; гипотеза 2] и [3; гипотеза 4.2].

Напомним, что действительное число $v$ называется алгебраически зависимым от действительных чисел $u_{1}, \ldots, u_{k}$, если $v$ удовлетворяет уравнению $p_{0}(u)+$ $p_{1}(u) v+\cdots+p_{n}(u) v^{n}=0$, где $p_{0}(u), \ldots, p_{n}(u)$ - полиномы от $u_{1}, \ldots, u_{k}$ с рациональньми коэффициентами, не все из которых являются нулевыми. Конечное

Работа вьполнена при финансовой поддержке Российского фонда фундаментальных исследований (грант № 03-01-00706) и NSERC (грант № 261914-03). 
множество действительных чисел называется алгебраически независимым, если ни одно из них не является алгебраически зависимьм от остальных.

СЛЕДСТВИЕ 1.2. Пусть $K$ - конечный симплициальный комплекс, $\theta: K \rightarrow \mathbb{R}^{m}$ - кусочно линейное отображсение $u \varepsilon>0$. Тогда существует такое кусочно линейное отображение $g: K \rightarrow \mathbb{R}^{m}$, что $d(g(v), \theta(v))<\varepsilon$ для всякой вершинь $v$ комплекса $K$ и для всяких иельх чисел $n, d, t, T$ $c 0 \leqslant t \leqslant d \leqslant T \leqslant m u d \leqslant m-n-1$ и всякой $d$-мерной плоскости $\Pi^{d} \subset \mathbb{R}^{m}$, параллельной некоторымм координатным плоскостям $\Pi^{t} \subset \Pi^{T} \subset \mathbb{R}^{m}$, число $q$ попарно дизбюнктных симплексов $K$ размерности $\leqslant n$, образы которых при $g$ пересекают $\Pi^{d}$, удовлетворяет неравенствам

$$
\begin{array}{ll}
q \leqslant d+1-t+\frac{n+(n+T-m)(d-t)}{m-n-d} & \text { npu } n \geqslant(m-n-T)(d-t), \\
q \leqslant 1+\frac{n}{m-n-T} & \text { npu } n \leqslant(m-n-T)(d-t) .
\end{array}
$$

Идея использования алгебраически независимых множеств для доказательства теорем общего положения, подобных следствию 1.2, восходит к Робертсу [4]. Эта идея применялась также Берковицем и Роем в работе [5], где они дали вариант следствия 1.2 при $t=0$ и $T=m$. Доказательство теоремы Берковица-Роя предложил Гудселл [6; теорема А.1] (в [7] даны другие приложения теоремы Берковица-Роя).

Приведем теперь некоторые приложения следствия 1.2 .

ТеОрема 1.3. Пусть $f: X \rightarrow Y$ - такое совериенное отображсение паракомпактных пространств, что $\operatorname{dim} f \leqslant n u \operatorname{dim} Y=0$. Тогда для всякого $m \geqslant n+1$ пространство $C^{*}\left(X, \mathbb{R}^{m}\right)$ всех ограниченных непрерывных отображений $X$ в $\mathbb{R}^{n}$ содержит плотное $G_{\delta}$-подмножество $\mathscr{H}$ таких отображений $g$, что $\operatorname{dim} g\left(f^{-1}(y)\right) \cap \Pi^{d} \leqslant n+d-m$ для всякой точки $y \in Y$ и всякой $d$-мерной плоскости $\Pi^{d} \subset \mathbb{R}^{m}$ с $m-n \leqslant d \leqslant m$.

СЛЕДСТВИЕ 1.4. Пусть $X$ - нормальное пространство с $\operatorname{dim} X \leqslant n u$ $m \geqslant n+1$. Тогда пространство $C^{*}\left(X, \mathbb{R}^{m}\right)$ в топологии равномерной сходимости содержит плотное $G_{\delta}$-подмножсество $\mathscr{H}$, состоящее из таких отобрахсений $g$, что пересечение $\overline{g(X)} \cap \Pi^{d}$ является не более чем $(n+d-m)$-мерным для всякой $d$-мерной плоскости $\Pi^{d} \subset \mathbb{R}^{m}$ с $m-n \leqslant d \leqslant m$.

Покажем, как следствие 1.4 вытекает из теоремы 1.3. Рассматривая стоун-чеховскую компактификацию $\beta X$ пространства $X$ и функциональное пространство $C\left(\beta X, \mathbb{R}^{m}\right)$ вместо $X$ и $C^{*}\left(X, \mathbb{R}^{m}\right)$ соответственно, мы можем предполагать, что $X$ компактно. К постоянному отображению $f$ на $X$ применим теорему 1.3.

Робертс доказал [4; теорема 1.2], что если $X$ - компактное метризуемое пространство размерности $\leqslant n$ и $n+1 \leqslant d \leqslant 2 n+1$, то $C\left(X, \mathbb{R}^{2 n+1}\right)$ в топологии равномерной сходимости содержит плотное $G_{\delta}$-подмножество, состоящее из таких отображений $g$, что $\operatorname{dim} g(X) \cap \Pi^{d} \leqslant d-n-1$ для всякой $d$-мерной плоскости $\Pi^{d} \subset \mathbb{R}^{2 n+1}$. С помощью этого результата и теоремы Гуревича о сохраняюшей размерность метризуемой компактификации Робертс вывел сушествование таких вложений для сепарабельных метризуемых пространств размерности $\leqslant n$. Очевидно, что результаты Робертса вытекают из следствия 1.4 и теоремы вложения Нёбелинга-Понтрягина. 
При $d \leqslant m-n-1$ теорема 1.3 не работает. Но следуюшая теорема показывает, что в этом случае мы можем доказать несколько больше: мы найдем в $C^{*}\left(X, \mathbb{R}^{m}\right)$ массивное множество, состояшее из таких отображений $g$, что пересечение $g\left(f^{-1}(y)\right) \cap \Pi^{d}$ конечно для всякой точки $y \in Y$ и всякой $d$-мерной плоскости в $\mathbb{R}^{m}$.

ТеОРема 1.5. Пусть $f: X \rightarrow Y$ - совершенное отображсение метризуемых пространств $c \operatorname{dim} f \leqslant n u \operatorname{dim} Y \leqslant 0$. Тогда пространство $C^{*}\left(X, \mathbb{R}^{m}\right)$ содержит плотное $G_{\delta}$-подмножество $\mathscr{K}$ таких отображений $g$, что для всяких чельх чисел $d, t, T c 0 \leqslant t \leqslant d \leqslant T \leqslant m u d \leqslant m-n-1$ и всякой $d$-мерной плоскости $\Pi^{d} \subset \mathbb{R}^{m}$, параллельной некоторьим координатнымм плоскостям $\Pi^{t} \subset \Pi^{T} \subset \mathbb{R}^{m}$, всякое мнохсество $f^{-1}(y) \cap g^{-1}\left(\Pi^{d}\right), y \in Y$, состоит из не более чем $q$ точек, где

$$
\begin{array}{ll}
q=d+1-t+\frac{n+(n+T-m)(d-t)}{m-n-d} & \text { nри } n \geqslant(m-n-T)(d-t), \\
q=1+\frac{n}{m-n-T} & \text { в противном случае. }
\end{array}
$$

СлЕДСТВИЕ 1.6. Пусть $X$ является метризуемым компактом $c \operatorname{dim} X \leqslant n$ $u m \geqslant n+1$. Тогда пространство $C\left(X, \mathbb{R}^{m}\right)$ содерәсит плотное $G_{\delta}$-подмножество, состоящее из таких отображений $g$, что для всяких чельх чисел $d, t, T c 0 \leqslant t \leqslant d \leqslant T \leqslant m u d \leqslant m-n-1$ и всякой $d$-мерной плоскости $\Pi^{d} \subset \mathbb{R}^{m}$, параллельной некоторым координатным плоскостям $\Pi^{t} \subset \Pi^{T}$ в $\mathbb{R}^{m}$, обратный прообраз $g^{-1}\left(\Pi^{d}\right)$ состоит не более чем из $q$ точек, где

$$
\begin{array}{ll}
q=d+1-t+\frac{n+(n+T-m)(d-t)}{m-n-d} & \text { nри } n \geqslant(m-n-T)(d-t), \\
q=1+\frac{n}{m-n-T} & \text { в противном случае. }
\end{array}
$$

Если в теореме 1.5 рассмотреть отображение в одноточечное пространство $Y$ при $m=n+2, d=1, t=0, T=r$, то получается

СлеДСТВИЕ 1.7. Пусть $X$ является метризуемым компактом $c \operatorname{dim} X \leqslant n$. Тогда пространство $C\left(X, \mathbb{R}^{n+2}\right)$ содержит плотное $G_{\delta}$-подмножсество, состоящее из таких отображений $g$, что для всякого натурального числа $r$ c $r \leqslant n+2$ и всякой прямой $\Pi^{1} \subset \mathbb{R}^{m}$, параллельной некоторой координатной r-мерной плоскости $\Pi^{r}$ в $\mathbb{R}^{m}$, обратный прообраз $g^{-1}\left(\Pi^{1}\right)$ состоит не более чем из $n+r$ точек.

Получаемые в настояшей работе отображения никогда не являются кусочно линейными, так как на всякой прямой лежит конечное число точек образа. Однако при $m \geqslant 2 n+1$ получаются вложения, причем ручные [8; теорема 5.1].

Нижеследующая теорема 1.8 является бесконечномерной версией теоремы 1.5. В случае компактного пространства $X$ и одноточечного пространства $Y$ теорема 1.8 была установлена Болтянским [9] при дополнительном ограничении $r=0$. 
Теорема 1.8. Пусть $f: X \rightarrow Y$ - совериенное отображение метризуемых пространств и $Y$ является $C$-пространством. Для чельх чисел $d, r$ пусть $\mathscr{P}(d, r)$ обозначает семейство всех $d$-мерньх плоскостей $\Pi^{d} \subset l_{2}$, параллельных некоторым координатным плоскостям $\Pi^{r} \subset l_{2}$. Тогда пространство $C^{*}\left(X, l_{2}\right)$ содержит плотное $G_{\delta}$-подмножсество таких отображений $g$, что для всякой точки $y \in Y$ и всякой плоскости $\Pi^{d} \in \mathscr{P}(d, r)$ множество $f^{-1}(y) \cap g^{-1}\left(\Pi^{d}\right)$ имеет не более $d+1-r$ точек при $r \leqslant d$ ине более одной точки при $r \geqslant d$.

Настоящая статья построена следующим образом. Доказательства теоремы 1.1 и следствия 1.2 даются в $\S 2$. Параграф̆ 3 посвящен доказательству теоремы 1.3. Теоремы 1.5 и 1.8 рассматриваются в $\S 4$. Последний $\S 5$ содержит дополнительные приложения теоремы 1.1 и следствия 1.2. В последний параграф включены также некоторые гипотезы.

Несколько слов по поводу основной предельной топологии [1]. Для любых пространств $M$ и $K$ через $C(K, M)$ мы обозначаем множество всех непрерывных отображений $K$ в $M$. Если $(M, d)$ - метрическое пространство и $K$ - произвольное пространство, то основная предельная топология на $C(K, M)$ определяется следующим образом: подмножество $U \subset C(K, M)$ открыто в $C(K, M)$ по отношению к основной предельной топологии, если для всякого отображения $g \in U$ существует такая непрерывная функция $\alpha: K \rightarrow(0, \infty)$, что $\bar{B}(g, \alpha) \subset U$. Здесь $\bar{B}(g, \alpha)$ обозначает множество $\{h \in C(K, M): d(g(x), h(x)) \leqslant \alpha(x)$ для всякой точки $x \in K\}$. Известно (см., например, [10]), что если $(M, d)$ является полньм метрическим пространством, то $C(K, M)$ в данной топологии обладает свойством Бэра. Это влечет, что пространство $C^{*}(K, H)$ в основной предельной топологии также обладает свойством Бэра для всякого банахова пространства $H$.

Результаты настоящей работы были получены во время визита первого автора в мае 2004 года в Ниписсинг университет. Автор благодарит NSERC за поддержку его визита и COMA Department Ниписсинг университета за гостеприимство.

В заключение авторы благодарят Т. Гудселла, предоставившего им свое доказательство [6; приложение] теоремы Берковица-Роя.

\section{§ 2. Доказательство теоремы 1.1 и следствия 1.2}

ДОКАЗАТЕЛЬСТво тЕОРЕМЫ 1.1. Предположим, что плоскость $\Pi^{d}$ пересекает линейную оболочку П $\left(M_{i}\right)$ всякого множества $M_{i}$, и пусть $Y_{i} \in \Pi^{d} \cap \Pi\left(M_{i}\right), i=$ $1, \ldots, q$. Достаточно показать, что в указанных предположениях или $n_{1}+n_{2}+$ $\cdots+n_{q}+1>(m-d)(q-1)-(T-d)(d-t)$ при $q \geqslant d-t+1$, или $n_{1}+$ $n_{2}+\cdots+n_{q}+1>(m-T)(q-1)$ при $1 \leqslant q \leqslant d-t+1$. Для дальнейшего нам необходимо нижеприводимое неравенство (см. [5] и [6]), которое вытекает из свойств алгебраически независимых множеств.

ПрЕДЛОЖЕНИЕ 2.1. Пусть дано алгебраччески независимое множество $A \subset \mathbb{R}$, и пусть множество $B \subset \mathbb{R}$ таково, что всякий элемент $A$ алгебраически зависит от множсества В. Тогда мощность $А$ не превосходит мощности $B$.

Нам необходимы также следующие построения Робертса [4]. Пусть $\left\{r_{i}\right\}$ - бесконечное алгебраически независимое множество, т.е. всякое конечное подмножество алгебраически независимо. Пусть $R_{i}=\left\{q+r_{i}: q \in \mathbb{Q}\right\}$, где $\mathbb{Q}$ - множество рациональных чисел. Тогда всякое множество $R_{i}$ плотно в $\mathbb{R}$ и различные множества $R_{i}$ 
дизъюнктны. Более того, всякое конечное множество $M$, содержащее не более одного числа из всякого $R_{i}, i=1,2, \ldots$, алгебраически независимо.

Мы можем предполагать, что $\Pi^{t}$ и $\Pi^{T}$ заданы первьми $t$ и $T$ координатами соответственно. Пусть $\pi$ - проекция $\mathbb{R}^{m}$ на пространство $\mathbb{R}^{m-t}$, заданное последними $m-t$ координатами. Тогда $\Pi^{d-t}=\pi\left(\Pi^{d}\right)$ является $(d-t)$-мерной плоскостью в $\mathbb{R}^{m-t}$, параллельной координатной плоскости $\Pi^{T-t}=\pi\left(\Pi^{T}\right)$. Кроме того, множество всех координат всех точек $B_{i, j}=\pi\left(A_{i, j}\right)$ алгебраически независимо (как подмножество координат точек $\left.A_{i, j}\right)$. Следовательно, рассматривая пространство $\mathbb{R}^{m-t}$, заданное последними $m-t$ координатами, и проекции $B_{i, j}, \pi\left(\Pi^{d}\right)$ и $\pi\left(\Pi^{T}\right)$ в это пространство, мы можем предполагать, что $t=0$.

Так как $Y_{i} \in \Pi\left(M_{i}\right)$, то сушествуют такие числа $\left\{\lambda_{i, j}\right\}_{j=1}^{n_{i}+1}$, что $\lambda_{i, 1}+\cdots+$ $\lambda_{i, n_{i}+1}=1$ и

$$
\begin{aligned}
& Y_{i}= \lambda_{i, 1} A_{i, 1}+\cdots+\lambda_{i, n_{i}} A_{i, n_{i}}+\lambda_{i, n_{i}+1} A_{i, n_{i}+1} \\
&= \lambda_{i, 1} A_{i, 1}+\cdots+\lambda_{i, n_{i}} A_{i, n_{i}}+\left(1-\lambda_{i, 1}-\cdots-\lambda_{i, n_{i}}\right) A_{i, n_{i}+1} \\
& \text { для всех } i=1, \ldots, q .
\end{aligned}
$$

По крайней мере одно из чисел $\left\{\lambda_{i, j}\right\}_{j=1}^{n_{i}+1}$ отлично от нуля. Без ограничения общности мы можем предполагать, что $\lambda_{i, 1} \neq 0$. (Если некоторые из чисел $\left\{\lambda_{i, j}\right\}_{j=1}^{n_{i}+1}$ являются нулевыми, то можно не рассматривать соответствующие точки и получить подмножество точек $\left\{A_{i, j}\right\}$, для которого предположения теоремы верны.) Из равенства (1) мы выразим точку $A_{i, 1}$ через точки $A_{i, 2}, \ldots, A_{i, n_{i}+1}, Y_{i}$. Тут возникает дополнительно $n_{i}$ чисел: $\lambda_{i, 1}, \ldots, \lambda_{i, n_{i}}$.

Мы выразили все координаты точек $\left\{A_{i, j}\right\}, i=1, \ldots, q, j=1, \ldots, n_{i}+1$, через координаты точек $\left\{A_{i, j}\right\}, i=1, \ldots, q, j=2, \ldots, n_{i}+1 ; Y_{1}, \ldots, Y_{q}$ и числа $\left\{\lambda_{i, j}\right\}$, $i=1, \ldots, q, j=1, \ldots, n_{i}$.

I. Пусть $q \leqslant d+1$. Так как плоскость $\Pi^{d}$ параллельна плоскости $\Pi^{T}$, то плоскость $Y_{1}+\Pi^{T}$ содержит все точки $Y_{2}, \ldots, Y_{q}$. Таким образом,

$$
Y_{i}-Y_{1}=\sum_{j=1}^{T} \alpha_{i, j} \mathbf{e}_{j}, \quad i=2, \ldots, q
$$

где $\mathbf{e}_{j}$ обозначает $j$-й единичный координатный вектор.

Мы выразили все координаты точек $\left\{A_{i, j}\right\}, i=1, \ldots, q, j=2, \ldots, n_{i}+1$; $Y_{1}, \ldots, Y_{q}$ и числа $\left\{\lambda_{i, j}\right\}, i=1, \ldots, q, j=1, \ldots, n_{i}$, через координаты точек $\left\{A_{i, j}\right\}$, $i=1, \ldots, q, j=2, \ldots, n_{i}+1 ; Y_{1}$ и числа $\left\{\lambda_{i, j}\right\}, i=1, \ldots, q, j=1, \ldots, n_{i}$; $\alpha_{i, j}, i=2, \ldots, q, j=1, \ldots, T$.

Следовательно, согласно предложению $2.1 q m \leqslant m+n_{1}+\cdots+n_{q}+T(q-1)$, т.е.

$$
n_{1}+\cdots+n_{q} \geqslant(m-T)(q-1) .
$$

Заметим, что ( $\left.3_{\mathrm{I}}\right)$ не меняется при замене $m, d$ и $T$ на $m-t, d-t$ и $T-t$ соответственно. Следовательно, (3I ) остается справедливым для всякого $t$ с $0 \leqslant t \leqslant d$.

II. Пусть $q \geqslant d+1$. Тогда существуют такие $d+1$ точка $Y_{i}$, скажем $Y_{1}, \ldots, Y_{d+1}$, что всякая точка $Y_{j}, j=d+2, \ldots, q$, является линейной комбинацией точек $Y_{i}$, $i=1, \ldots, d+1$. Отметим, что такие $d+1$ точка существуют, даже если линейная оболочка всех точек $Y_{1}, \ldots, Y_{q}$ имеет размерность $<d$. Следовательно,

$$
Y_{j}=\sum_{i=1}^{d} \beta_{j, i} Y_{i}+\left(1-\sum_{i=1}^{d} \beta_{j, i}\right) Y_{d+1}, \quad j=d+2, \ldots, q,
$$


для некоторых чисел $\left\{\beta_{j, i}\right\}, i=1, \ldots, d, j=d+2, \ldots, q$.

В этом случае уравнения (2) верны для $i=2, \ldots, d+1$. Таким образом, мы выразили все координаты точек $\left\{A_{i, j}\right\}, i=1, \ldots, q, j=2, \ldots, n_{i}+1 ; Y_{1}, \ldots, Y_{q}$ и числа $\left\{\lambda_{i, j}\right\}, i=1, \ldots, q, j=1, \ldots, n_{i}$, через координаты точек $\left\{A_{i, j}\right\}, i=$ $1, \ldots, q, j=2, \ldots, n_{i}+1 ; Y_{1}$ и числа $\left\{\lambda_{i, j}\right\}, i=1, \ldots, q, j=1, \ldots, n_{i} ;\left\{\beta_{j, i}\right\}$, $j=d+2, \ldots, q, i=1, \ldots, d ; \alpha_{i, j}, i=2, \ldots, d+1, j=1, \ldots, T$.

Следовательно, согласно предложению $2.1 q m \leqslant m+n_{1}+\cdots+n_{q}+(q-d-1) d+$ $T d$, т.е. $n_{1}+\cdots+n_{q} \geqslant(m-d)(q-1)-(T-d) d$. Заменяя в последнем неравенстве $m, d$ и $T$ на $m-t, d-t$ и $T-t$ соответственно, мы получаем неравенство

$$
n_{1}+\cdots+n_{q} \geqslant(m-d)(q-1)-(T-d)(d-t) .
$$

Неравенства (3I ) и (3II) завершают доказательство теоремы 1.1.

ДокАЗАТЕЛЬСТво СЛЕДСТВИя 1.2. Пусть $\left\{v_{i}\right\}$ обозначают вершины комплекса $K$ и $R_{j}$ - множества из обсуждавшегося выше построения Робертса, проведенного для некоторого счетного алгебраически независимого множества. Для всякого $i$ выберем такую точку $A_{i}=\left(A_{i}(1), \ldots, A_{i}(m)\right) \in \mathbb{R}^{m}$, что $\operatorname{dist}\left(\theta\left(v_{i}\right), A_{i}\right)<\varepsilon$ и $A_{i}(s) \in R_{(i-1) m+s}$. Тогда множество всех координат $\left\{A_{i}(k)\right\}$ алгебраически независимо. Определим отображение $g: K \rightarrow \mathbb{R}^{m}$ по формуле $g\left(v_{i}\right)=A_{i}$ и продолжим далее $g$ линейно на все симплексы $K$. Очевидно, что $g\left(v_{i_{1}}\right) \neq g\left(v_{i_{2}}\right)$ при $i_{1} \neq i_{2}$. Кроме того, ограничение отображения $g$ на всякий $n$-мерный симплекс $K$ является взаимно однозначньм. Пусть $\Pi^{d}$ является $d$-мерной плоскостью в $R^{m}$, параллельной некоторым координатным плоскостям $\Pi^{t} \subset \Pi^{T}$, и $q$ - число попарно дизъюнктных не более чем $n$-мерных симплексов $K$, образы которых при $g$ пересекают $\Pi^{d}$. Итак, пусть в $K$ существуют $q$ не более чем $n$-мерных попарно дизъюнктных симплексов $\sigma_{i}=\left\langle v_{i, j}: j=1, \ldots, n_{i}+1\right\rangle$, образы которых $g\left(\sigma_{i}\right)=\left\langle A_{i, j}: j=1, \ldots, n_{i}+1\right\rangle$ пересекают $\Pi^{d}$.

Покажем, что

$$
q \leqslant N_{1}=d+1-t+\frac{n+(n+T-m)(d-t)}{m-n-d}, \text { если } n \geqslant(m-n-T)(d-t) .
$$

Предположим, что $q \geqslant d+1-t$. Так как $\Pi^{d}$ пересекает образы $g\left(\sigma_{i}\right) \subset \Pi\left(\left\{A_{i, j}\right.\right.$ : $\left.\left.j=1, \ldots, n_{i}+1\right\}\right)$ всех непересекающихся симплексов $\sigma_{i}, i=1, \ldots, q$, то согласно теореме $1.1 n_{1}+\cdots+n_{q} \geqslant(m-d)(q-1)-(T-d)(d-t)$. Следовательно,

$$
n q \geqslant(m-d)(q-1)-(T-d)(d-t),
$$

так как $n_{i} \leqslant n$ для всех $i$. Полученное неравенство (4) завершает доказательство нужного неравенства $q \leqslant N_{1}$.

Если же $q \leqslant d+1-t$, то неравенство $q \leqslant N_{1}$ справедливо, так как согласно нашему предположению $n \geqslant(m-n-T)(d-t)$, т.е. $d+1-t \leqslant N_{1}$.

Теперь покажем, что

$$
q \leqslant N_{2}=1+\frac{n}{m-n-T}, \text { если } n \leqslant(m-n-T)(d-t) .
$$

Предположим, что $q \leqslant d+1-t$. Тогда, как и вьше, теорема 1.1 влечет неравенство $n_{1}+\cdots+n_{q} \geqslant(m-T)(q-1)$. Следовательно,

$$
n q \geqslant(m-T)(q-1)
$$


так как $n_{i} \leqslant n$ для всех $i$. Полученное неравенство (5) завершает доказательство нужного неравенства $q \leqslant N_{2}$.

Если же $q \geqslant d-t+1$, то, как уже было доказано выше, имеет место неравенство $q \leqslant N_{1}$. Согласно же нашему предположению $n \leqslant(m-n-T)(d-t)$, т.е. $d+1-t \leqslant N_{1}$. Полученные неравенства означают, что при сделанных предположениях $q=d-t+1=N_{1}$ и $n=(m-n-T)(d-t)$, поэтому $q=d-t+1=N_{1}=N_{2}$.

\section{§3. Доказательство теоремы 1.3}

Сначала докажем теорему 1.3 в специальном случае $d=m-n$. Итак, мы фиксируем пространства $X, Y$ и отображение $f$, удовлетворяющие предположениям теоремы 1.3. Если не указано обратное, то в этом параграфе мы пользуемся следующими обозначениями: $\varrho$ обозначает евклидову метрику в $\mathbb{R}^{m}, \varepsilon$-дизъюнктное множество $\mathbb{R}^{m}$ - это подмножество, которое покрывается семейством открытых дизъюнктных подмножеств $\mathbb{R}^{m}$, каждое из которых имеет диаметр $<\varepsilon$. Мы говорим, что подмножество $A \subset \mathbb{R}^{m}$ имеет тип $(d, \varepsilon)$, если множество $\Pi^{d} \cap A$ является $\varepsilon$-дизъюнктным для всякой $d$-плоскости $\Pi^{d}$ в $\mathbb{R}^{m}$.

Пусть $\mathscr{H}_{\varepsilon}, \varepsilon>0$, обозначает множество таких отображений $g \in C^{*}\left(X, \mathbb{R}^{m}\right)$, что $g\left(f^{-1}(y)\right)$ имеет тип $(m-n, \varepsilon)$ для всякой точки $y \in Y$. Так как пространство $C^{*}\left(X, \mathbb{R}^{m}\right)$ в основной предельной топологии обладает свойством Бэра, то достаточно показать, что всякое множество $\mathscr{H}_{\varepsilon}$ открыто и плотно в $C^{*}\left(X, \mathbb{R}^{m}\right)$. Действительно, тогда множество $\mathscr{H}=\bigcap_{k=1}^{\infty} \mathscr{H}_{1 / k}$ будет плотным и иметь тип $G_{\delta}$ в $C^{*}\left(X, \mathbb{R}^{m}\right)$. При этом если $g \in \mathscr{H}$ и $y \in Y$, то $g\left(f^{-1}(y)\right) \cap \Pi$ не более чем 0-мерно для всякой $(n-m)$-плоскости $\Pi \subset \mathbb{R}^{m}$.

Лемма 3.1. Пусть $A \subset X$ компактно $и \varepsilon>0$. Пусть также для некотоpьх $g_{0} \in C^{*}\left(X, \mathbb{R}^{m}\right)$ и $d$ множество $g_{0}(A)$ имеет тип $(d, \varepsilon)$. Тогда существуют такие окрестность $U$ множества $A$ в $X$ и $\delta>0$, что множество $\overline{g(U)}$ имеет тип $(d, \varepsilon)$ для всякого такого отобрахсения $g \in C^{*}\left(X, \mathbb{R}^{m}\right)$, что $g \mid U$ $u g_{0} \mid U \quad \delta$-близки.

ДокАЗАТЕЛьство. Предположим, что заключение леммы неверно. Для получения противоречия мы будем следовать доказательству п. 2.4 в [4; с. 569]. Для всякого $i \geqslant 1$ возьмем такую окрестность $U_{i}$ множества $A$, что $U_{i} \subset g_{0}^{-1}\left(W_{i}\right)$, где $W_{i}$ является $1 / i$-окрестностью $g_{0}(A)$. Существуют такие отображение $g_{i} \in$ $C^{*}\left(X, \mathbb{R}^{m}\right)$ и $d$-плоскость $\Pi_{i}^{d}$, что $g_{i} \mid U_{i}$ и $g_{0} \mid U_{i} 1 / i$-близки, но множество $\overline{g_{i}\left(U_{i}\right)} \cap$ $\Pi_{i}^{d}$ не является $\varepsilon$-дизъюнктным. Выберем точки $z_{i} \in \overline{g_{i}\left(U_{i}\right)} \cap \Pi_{i}^{d}$ и $x_{i} \in U_{i}$ такие, что $\rho\left(g_{i}\left(x_{i}\right), z_{i}\right) \leqslant 1 / i, i \geqslant 1$. Очевидно, что $K=\left\{z_{i}\right\}_{i=1}^{\infty} \cup g_{0}(A)$ является компактом, пересекающим всякую плоскость $\Pi_{i}^{d}$. Следовательно, существует подпоследовательность $\left\{\Pi_{i}^{d}\right\}_{i=1}^{\infty}$, сходяшаяся к $d$-плоскости $\Pi_{0}^{d}$. Мы будем предполагать, что плоскости $\left\{\Pi_{i}^{d}\right\}_{i=1}^{\infty}$ сами сходятся к $\Pi_{0}^{d}$.

Пусть $V$ - открытое подмножество $\mathbb{R}^{m}$, содержащее $g_{0}(A) \cap \Pi_{0}^{d}$ и являюшееся объединением конечного семейства открытых дизъюнктных подмножеств $\mathbb{R}^{m}$ диаметра $<\varepsilon$. Так как всякое $\overline{g_{i}\left(U_{i}\right)} \cap \Pi_{i}^{d}$ не является $\varepsilon$-дизъюнктньм, то существуют такие точки $a_{i} \in U_{i}$ и $b_{i} \in \overline{g_{i}\left(U_{i}\right)} \cap \Pi_{i}^{d}$, что $V$ не содержит множество $\left\{g_{i}\left(a_{i}\right), b_{i}\right\}_{i=1}^{\infty}$. Мы можем также считать, что $\varrho\left(b_{i}, g_{i}\left(a_{i}\right)\right) \leqslant 1 / i$ для всех $i$. Это влечет сушествование точки $b \in g_{0}(A)$ и подпоследовательности $\left\{b_{i}\right\}$, сходящейся к $b$. Мы опять будем писать $\lim b_{i}=b$. Тогда $b \in \Pi_{0}^{d}$, так как $\left\{\Pi_{i}^{d}\right\}$ сходится к $\Pi_{0}^{d}$. Следовательно, $b \in g_{0}(A) \cap \Pi_{0}^{d} \subset V$. Поэтому $b_{i} \in V$ для некоторого $i$, что противоречит выбору $b_{i}$. 
СЛЕДСТВИЕ 3.2. Пусть $g_{0}\left(f^{-1}\left(y_{0}\right)\right)$ имеет тип $(m-n, \varepsilon)$ для некоторой точки $y_{0} \in Y$ и $g_{0} \in C^{*}\left(X, \mathbb{R}^{m}\right)$. Тогда существуют окрестность $V$ точки у в $Y$ и $\delta>0$ такие, что множество $\overline{g\left(f^{-1}(V)\right)}$ имеет тип $(m-n, \varepsilon)$ для всякого такого отобрахения $g \in C^{*}\left(X, \mathbb{R}^{m}\right)$, что $g \mid f^{-1}(V)$ и $g_{0} \mid f^{-1}(V)$

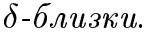

ДоКАЗАТЕЛЬСтво. Применяя лемму $3.1 \mathrm{k} f^{-1}\left(y_{0}\right)$, мы получаем $\delta>0$ и такую окрестность $U$ множества $f^{-1}\left(y_{0}\right)$, что множество $\overline{g(U)}$ имеет тип $(m-n, \varepsilon)$, как только $g \in C^{*}\left(X, \mathbb{R}^{m}\right)$ и $g \mid U \delta$-близко к $g_{0} \mid U$. Так как отображение $f$ замкнуто, то можно найти замкнутую окрестность $V$ точки $y_{0}$ в $Y$ такую, что $f^{-1}(V) \subset U$. Теперь для некоторого отображения $g \in C^{*}\left(X, \mathbb{R}^{m}\right)$ пусть $g \mid f^{-1}(V)$ и $g_{0} \mid f^{-1}(V)$ $\delta$-близки. Продолжим $g \mid f^{-1}(V)$ до такого отображения $h \in C^{*}\left(X, \mathbb{R}^{m}\right)$, что $h \mid U$ остается $\delta$-близким к $g_{0} \mid U$. Тогда согласно выбору $U$ и $\delta$ множество $\overline{h(U)}$ имеет тип $(m-n, \varepsilon)$. Теперь, так как $\overline{h\left(f^{-1}(V)\right)} \subset \overline{h(U)}$ и $h\left|f^{-1}(V)=g\right| f^{-1}(V)$, доказательство завершено.

ПРЕДЛОЖЕНИЕ 3.3. Множсество $\mathscr{H}_{\varepsilon}$ открыто в $C^{*}\left(X, \mathbb{R}^{m}\right)$.

ДокаЗАТЕЛЬство. Фиксируем $g_{0} \in \mathscr{H}_{\varepsilon}$. Согласно следствию 3.2 для всякой точки $y \in Y$ сушествуют такая окрестность $V_{y}$ в $Y$ и $\delta_{y}>0$, что если $g \in C^{*}\left(X, \mathbb{R}^{m}\right)$ и $\varrho\left(g(x), g_{0}(x)\right) \leqslant \delta_{y}$ для всех точек $x \in f^{-1}\left(V_{y}\right)$, то $\overline{g\left(f^{-1}\left(V_{y}\right)\right)}$ имеет тип $(m-n, \varepsilon)$. Возьмем локально конечное открытое покрытие $\omega$ пространства $Y$, вписанное в $\left\{V_{y}: y \in Y\right\}$, и для всякого $W \in \omega$ фиксируем такую точку $y(W) \in Y$, что $W \subset V_{y(W)}$. Определим многозначное отображение $\phi: Y \rightarrow(0, \infty)$ формулой $\phi(y)=\bigcup\left\{\left(0, \delta_{y(W)}\right]: y \in W\right\}$. Очевидно, что $\phi$ выпуклозначно и полунепрерывно снизу. Согласно [11; теорема 6.2] отображение $\phi$ допускает непрерывную селекцию $\beta: Y \rightarrow(0, \infty)$, и пусть $\alpha=\beta \circ f$. Достаточно показать, что из $g \in C^{*}\left(X, \mathbb{R}^{m}\right)$ и $\varrho\left(g_{0}(x), g(x)\right)<\alpha(x)$ для всякой точки $x \in X$ следует, что $g \in \mathscr{H}_{\varepsilon}$. Теперь пусть $y \in Y$ и выберем такой элемент $W \in \omega$, содержащий $y$, что $\alpha(x) \leqslant \delta_{y(W)}$ для всякой точки $x \in f^{-1}(y)$. Возьмем функцию $h_{y} \in C^{*}\left(X, \mathbb{R}^{m}\right)$, совпадающую с $g$ на множестве $f^{-1}(y)$ и удовлетворяющую неравенству $\varrho\left(h_{y}(x), g_{0}(x)\right) \leqslant \delta_{y(W)}$ для всех $x \in X$. Тогда в соответствии с выбором $V_{y(W)}$ множество $\overline{h_{y}\left(f^{-1}\left(V_{y(W)}\right)\right)}$, а значит, и множество $\overline{g\left(f^{-1}(y)\right)}$ имеет тип $(m-n, \varepsilon)$. Поэтому всякое отображение $g \in C^{*}\left(X, \mathbb{R}^{m}\right)$, которое $\alpha$-близко к $g_{0}$, принадлежит $\mathscr{H}_{\varepsilon}$. Следовательно, $\mathscr{H}_{\varepsilon}$ открыто в $C^{*}\left(X, \mathbb{R}^{m}\right)$.

Для пространства $M$ и $\varepsilon>0$ пусть $C_{(n, \varepsilon)}\left(M, \mathbb{R}^{m}\right)$ обозначает множество всех таких отображений $g \in C^{*}\left(M, \mathbb{R}^{m}\right)$, что $g(M)$ имеет тип $(m-n, \varepsilon)$ при $m \geqslant n+1$.

Лемма 3.4. Пусть $M-n$-мерный компакт и $m \geqslant n+1$. Тогда $C_{(n, \varepsilon)}\left(M, \mathbb{R}^{m}\right)$ плотно в $C\left(M, \mathbb{R}^{m}\right)$ для всякого $\varepsilon>0$.

ДокаЗАтЕЛьство. Пусть $g_{0} \in C\left(M, \mathbb{R}^{m}\right)$ и $\delta>0$. Представляя $g_{0}$ как композицию двух отображений $q_{1}: M \rightarrow Z$ и $q_{2}: Z \rightarrow \mathbb{R}^{m}$, где $Z$ - метризуемый компакт размерности $\leqslant n$, и рассматривая $Z$ и $q_{2}$ вместо $M$ и $g_{0}$, мы редуцируем наше доказательство к случаю метризуемого компакта $M$. Выберемположительное число $\eta$, удовлетворяющее следующим условиям:

$$
5 \eta<\frac{\delta}{2} \quad \text { и } 9 \eta(r+1)<\varepsilon, \text { где } r=n(m+1-n) .
$$


Так как $\operatorname{dim} M \leqslant n$, то стандартной процедурой мы можем найти конечный $n$-мерный комплекс $K$ и такие отображения $h: M \rightarrow K, \theta: K \rightarrow \mathbb{R}^{m}$, что $\theta \circ h \delta / 2$ близко к $g_{0}$. Более того, мы можем предполагать, что

$$
\operatorname{diam}(\theta(\sigma))<\eta \quad \text { для всякого симплекса } \sigma \in K .
$$

Достаточно найти отображение $g: K \rightarrow \mathbb{R}^{m}$, которое $\delta / 2$-близко к отображению $\theta$ и $g \circ h \in C_{(n, \varepsilon)}\left(M, \mathbb{R}^{m}\right)$. Для этого мы воспользуемся следствием 1.2 (c $d=m-n$, $t=0, T=m, \varepsilon=\eta$ и $n$, замененным на $n-1)$ для получения такого кусочно линейного отображения $g: K \rightarrow \mathbb{R}^{m}$, что $\varrho(g(v), \theta(v)) \leqslant \eta$ для всех вершин $v$ комплекса $K$ и для всякой $(m-n)$-плоскости $\Pi \subset \mathbb{R}^{m}$ число $q$ дизъюнктных не более чем $(n-1)$-мерных симплексов $K$, образы которых при $g$ пересекают П, не превосходит $r=n(m+1-n)$. Мы можем выбрать $g$ так, чтобы дополнительно $g\left(v_{i}\right) \neq g\left(v_{j}\right)$ для всяких различных вершин $v_{i}$ и $v_{j}$ комплекса $K$.

Пусть $v_{i}$ и $v_{j}-$ две вершины одного симплекса $\Delta \in K$. Тогда согласно (7) и выбору $g$ мы имеем

$$
\varrho\left(g\left(v_{i}\right), g\left(v_{j}\right)\right) \leqslant \varrho\left(g\left(v_{i}\right), \theta\left(v_{i}\right)\right)+\varrho\left(\theta\left(v_{i}\right), \theta\left(v_{j}\right)\right)+\varrho\left(\theta\left(v_{j}\right), g\left(v_{j}\right)\right)<3 \eta .
$$

Следовательно,

$$
g(\Delta) \text { имеет диаметр }<3 \eta \text { для всякого симплекса } \Delta \in K \text {. }
$$

Условие $(8)$ влечет, что $\varrho(g(y), \theta(y))<5 \eta$ для всех $y \in K$. Поэтому согласно (6) отображения $g$ и $\theta \delta / 2$-близки.

Осталось показать только, что $g \circ h \in C_{(n, \varepsilon)}\left(M, \mathbb{R}^{m}\right)$ или, эквивалентно, что $g(K)$ имеет тип $(m-n, \varepsilon)$. Для этого мы воспользуемся идеей из работы $[4 ;$ доказательство п. 2.3, с. 568]. Фиксируем $(m-n)$-плоскость $\Pi \subset \mathbb{R}^{m}$. Достаточно доказать, что всякая компонента множества $g(K) \cap$ П имеет диаметр $\leqslant 9(r+1) \eta$, так как $9(r+1) \eta<\varepsilon$ согласно (6). Предположим, что $\varrho(a, b)>9(r+1) \eta$ для некоторой компоненты $P$ множества $g(K) \cap \Pi$ и некоторых точек $a \in P, b \in P$. Возьмем в $P$ кривую $a b$. Согласно (8) всякая поддуга $a b$ диаметра $\geqslant 3 \eta$ должна содержать по крайней мере одну точку на границе симплекса $g(K)$, следовательно, на симплексе $g(K)$ размерности $\leqslant n-1$. Далее, выберем такие точки $a_{i} \in a b$, $i=1, \ldots, r+1$, что $3(3 i-2) \eta<\varrho\left(a, a_{i}\right) \leqslant 3(3 i-1) \eta, i=1, \ldots, r+1$, и всякая точка $a_{i}$ принадлежит симплексу $g\left(\sigma_{i}\right) \in g(K)$ размерности $\leqslant n-1$. Тогда $\varrho\left(a_{i}, a_{j}\right)>6 \eta$ для $i \neq j$, что согласно (8) влечет $g\left(\sigma_{i}\right) \cap g\left(\sigma_{j}\right)=\varnothing$. Следовательно, мы получили $r+1$ дизъюнктные симплексы $\sigma_{i} \in K, i=1, \ldots, r+1$, каждый размерности $\leqslant n-1$, образы которых при $g$ пересекают П. Получено противоречие, так как согласно выбору $g$ число попарно непересекающихся не более чем $(n-1)$-мерных симплексов $K$, образы которых при $g$ пересекают П, не превосходит $n(m-n+1)=r$.

Далее рассматриваем многозначное отображение $\psi_{\varepsilon}: Y \rightarrow C^{*}\left(X, \mathbb{R}^{m}\right)$, определенное формулой $\psi_{\varepsilon}(y)=C^{*}\left(X, \mathbb{R}^{m}\right) \backslash \mathscr{H}_{\varepsilon}(y)$, где $\mathscr{H}_{\varepsilon}(y)$ обозначает множество всех таких отображений $g \in C^{*}\left(X, \mathbb{R}^{m}\right)$, что $g\left(f^{-1}(y)\right)$ имеет тип $(m-n, \varepsilon)$.

Следующее предложение вместе с предложением 3.3 завершает доказательство теоремы 1.3 для $k=m-n$.

ПреДЛОЖЕНИЕ 3.5. Множество $\mathscr{H}_{\varepsilon}$ плотно в $C^{*}\left(X, \mathbb{R}^{m}\right)$. 
ДокАЗАТЕльство. Сначала покажем, что график $G$ отображения $\psi_{\varepsilon}$ замкнут в $Y \times C^{*}\left(X, \mathbb{R}^{m}\right)$, если $C^{*}\left(X, \mathbb{R}^{m}\right)$ наделено топологией равномерной сходимости, порожденной метрикой $\varrho$. Пусть $\left(y_{0}, g_{0}\right) \in\left(Y \times C^{*}\left(X, \mathbb{R}^{m}\right)\right) \backslash G$. Тогда $g_{0} \notin \psi_{\varepsilon}\left(y_{0}\right)$, поэтому $g_{0} \in \mathscr{H}_{\varepsilon}\left(y_{0}\right)$. Согласно следствию 3.2 существуют такие $\delta>0$ и окрестность $V$ точки $y_{0}$ в $Y$, что $\overline{g\left(f^{-1}(V)\right)}$ имеет тип $(m-n, \varepsilon)$ для всякого такого отображения $g \in C^{*}\left(X, \mathbb{R}^{m}\right)$, что $g \mid f^{-1}(V) \delta$-близко к $g_{0} \mid f^{-1}(V)$. Пусть $W \subset C^{*}\left(X, \mathbb{R}^{m}\right)$ - множество всех отображений $g$, которые $\delta$-близки к $g_{0}$. Очевидно, что $W$ является окрестностью $g_{0}$ в $C^{*}\left(X, \mathbb{R}^{m}\right)$ в топологии равномерной сходимости. Таким образом, $V \times W$ является окрестностью $\left(y_{0}, g_{0}\right)$ в $Y \times C^{*}\left(X, \mathbb{R}^{m}\right)$, которая не пересекает $G$. Следовательно, $G$ замкнуто в $Y \times C^{*}\left(X, \mathbb{R}^{m}\right)$.

УтВеРЖ ДЕНИЕ. $\bar{B}\left(g_{0}, \alpha\right) \backslash \psi_{\varepsilon}(y) \neq \varnothing$ для всякой точки $y \in Y, \alpha: X \rightarrow(0, \infty)$ $u g_{0} \in C^{*}\left(X, \mathbb{R}^{m}\right)$.

ДокаЗАТЕЛЬство. Нам необходимо показать, что $\bar{B}\left(g_{0}, \alpha\right) \cap \mathscr{H}_{\varepsilon}(y) \neq \varnothing$ для фиксированных $y \in Y, \alpha \in C(X,(0, \infty))$ и $g_{0} \in C^{*}\left(X, \mathbb{R}^{m}\right)$. Пусть $\delta>0$ - минимальное значение $\alpha$ на $f^{-1}(y)$. Так как $\operatorname{dim} f^{-1}(y) \leqslant n$, то по лемме 3.4 сушествует отображение $h \in C\left(f^{-1}(y), \mathbb{R}^{m}\right)$, которое $\delta$-близко к $g_{0} \mid f^{-1}(y)$ и такое, что $h\left(f^{-1}(y)\right)$ имеет тип $(m-n, \varepsilon)$. Очевидно, что $\varrho\left(h(x), g_{0}(x)\right) \leqslant \alpha(x)$ для всех $x \in f^{-1}(y)$. Таким образом, всякое продолжение $g \in C^{*}\left(X, \mathbb{R}^{m}\right)$ отображения $h$ лежит в $\mathscr{H}_{\varepsilon}(y)$. Следовательно, доказательство редуцировано к нахождению такого продолжения отображения $h$, которое лежит также в $\bar{B}\left(g_{0}, \alpha\right)$. Для этого мы определяем многозначное отображение $\Phi: X \rightarrow \mathbb{R}^{m}$ формулой $\Phi(x)=h(x)$ при $x \in f^{-1}(y)$ и $\Phi(x)=B\left(g_{0}(x), \alpha(x)\right)$ при $x \notin f^{-1}(y)$. Здесь $B\left(g_{0}(x), \alpha(x)\right)$ обозначает замкнутый шар в $\mathbb{R}^{m}$ с центром $g_{0}(x)$ и радиусом $\alpha(x)$. Это отображение полунепрерывно снизу с замкнутыми и выпукльми образами в $\mathbb{R}^{m}$. Поэтому согласно выпуклозначной селекционной теореме Майкла отображение $\Phi$ допускает непрерывную селекцию $g$, которая продолжает $h$. Кроме того, мы можем считать, что $\alpha$ является ограниченной функцией, что влечет ограниченность $g$. Таким обpaзом, $g \in \bar{B}\left(g_{0}, \alpha\right)$, что завершает доказательство утверждения.

Теперь мы в состоянии завершить доказательство нашего предложения. Фиксируем $g_{0} \in C^{*}\left(X, \mathbb{R}^{m}\right)$ и $\alpha \in C^{*}(X,(0,1))$ и рассмотрим постоянное многозначное отображение $\varphi: Y \rightarrow C^{*}\left(X, \mathbb{R}^{m}\right), \varphi(y)=\bar{B}\left(g_{0}, \alpha\right)$, где $C^{*}\left(X, \mathbb{R}^{m}\right)$ наделяется топологией равномерной сходимости. Нам понадобится следующая теорема Майкла [12; теорема 5.3]:

Пусть $Y$ - паракомпактное пространство и $\operatorname{dim} Y=0, M-$ полное метризуемое пространство, а $\phi: Y \rightarrow M$ - полунепрерывное снизу замкнутозначное отображение. Если $\psi: Y \rightarrow M-$ такое многозначное отображсение с замкнутым графиком, что $\phi(y) \backslash \psi(y) \neq \varnothing$ для всех точек $y \in Y$, то ф имеет селекиию, избегающую $\psi$.

В нашем случае $\varphi$ и $\psi_{\varepsilon}$ удовлетворяют предположениям теоремы Майкла, поэтому существует такое отображение $\theta: Y \rightarrow C^{*}\left(X, \mathbb{R}^{m}\right)$, что $\theta(y) \in \bar{B}\left(g_{0}, \alpha\right) \backslash$ $\psi_{\varepsilon}(y)$ для всех точек $y \in Y$. Отображение $g \in C^{*}\left(X, \mathbb{R}^{m}\right)$ определим формулой $g(x)=\theta(f(x)), x \in X$. Тогда $g \in \bar{B}\left(g_{0}, \alpha\right) \cap \mathscr{H}_{\varepsilon}(y)$ для всякой точки $y \in Y$. Следовательно, $g \in \bar{B}\left(g_{0}, \alpha\right) \cap \mathscr{H}_{\varepsilon}$ и доказательство завершено.

Как мы уже указывали, из предложения 3.3 и предложения 3.5 следует, что множество $\mathscr{H}$ плотно и имеет тип $G_{\delta}$ в $C^{*}\left(X, \mathbb{R}^{m}\right)$. Это дает доказательство теоремы 1.3 в специальном случае $d=m-n$. Докажем теперь общий случай теоремы 1.3. Мы покажем, что всякое отображение $g \in \mathscr{H}$ удовлетворяет теореме, т.е. 
удовлетворяет следующему условию: компакт $A=g\left(f^{-1}(y)\right) \cap \Pi^{d}$ не более чем $(n+d-m)$-мерен для всякой точки $y \in Y$ и всякой $d$-плоскости $\Pi^{d} \subset \mathbb{R}^{m}$ при $m-n+1 \leqslant d \leqslant m$. Фиксируем некоторую $(m-n)$-плоскость $\Pi^{m-n} \subset \Pi^{d}$ и рассмотрим ортогональную проекцию $p$ плоскости $\Pi^{d}$ на $(n+d-m)$-плоскость $\Pi^{n+d-m} \subset \Pi^{d}-$ ортогональное дополнение плоскости $\Pi^{m-n}$ в $\Pi^{d}$. Тогда компакт $B=p\left(g\left(f^{-1}(y)\right) \cap \Pi^{d}\right) \subset \Pi^{n+d-m}$ имеет размерность $\leqslant n+d-m$. Так как слоями отображения $\left.p\right|_{A}: A \rightarrow B$ являются пересечения компакта $g\left(f^{-1}(y)\right)$ c $(m-n)$-плоскостями, то согласно выбору отображения $g$ эти слои нульмерны. Следовательно, согласно теореме Гуревича о нульмерных совершенных отображениях имеет место искомое неравенство $\operatorname{dim} A \leqslant \operatorname{dim} B \leqslant n+d-m$.

\section{§4. Доказательство теоремы 1.5 и теоремы 1.8}

ДокАЗАТЕЛЬСтво теоРемЫ 1.5. Достаточно доказать, что для всяких целых чисел $d, t, T$ таких, что $0 \leqslant t \leqslant d \leqslant T \leqslant m$ и $d \leqslant m-n-1$, и всяких координатных плоскостей $\Pi^{t} \subset \Pi^{T}$ в $\mathbb{R}^{m}$ пространство $C^{*}\left(X, \mathbb{R}^{m}\right)$ содержит плотное $G_{\delta}$-подмножество таких отображений $g$, что множество $f^{-1}(y) \cap g^{-1}\left(\Pi^{d}\right)$ состоит из не более чем $q$ точек для всякой точки $y \in Y$ и всякой $d$-плоскости $\Pi^{d} \subset \mathbb{R}^{m}$, параллельной $\Pi^{t} \subset \Pi^{T}$, где $q$ - это целая часть $N_{1}=d+1-t+$ $\frac{n+(n+T-m)(d-t)}{n-n-d}$ при $n \geqslant(m-n-T)(d-t)$ и целая часть $N_{2}=1+$ $\frac{n}{m-n-T}$ в противном случае. Таким образом, мы фиксируем целые числа $d$, $t, T$, удовлетворяюшие вышеприведенньм неравенствам, и координатные плоскости $\Pi^{t} \subset \Pi^{T} \subset \mathbb{R}^{m}$. Подчеркнем, что всегда $q \geqslant 1$, и пусть $\mathscr{P}$ - это множество всех $d$-плоскостей в $\mathbb{R}^{m}$, параллельных $\Pi^{t} \subset \Pi^{T}$. Теперь мы определим следующее понятие, которым пользуемся в этом параграфе: подмножество $A$ произвольного метрического пространства $M$ назьвается имеющим $\operatorname{komun}(q, \varepsilon, g)$, где $\varepsilon>0$ и $g \in C^{*}\left(M, \mathbb{R}^{m}\right)$, если для всякой плоскости $\Pi^{d} \in \mathscr{P}$ множество $A \cap g^{-1}\left(\Pi^{d}\right)$ можно покрыть не более чем $q$ дизъюнктньми открытыми подмножествами $M$ диаметра $\leqslant \varepsilon$. Пусть $\mathscr{K}_{\varepsilon}, \varepsilon>0$, обозначает множество всех таких отображений $g \in C^{*}\left(X, \mathbb{R}^{m}\right)$, что $f^{-1}(y)$ имеет котип $(q, \varepsilon, g)$ для всякой точки $y \in Y$. Доказательство редуцировано к проверке, что всякое множество $\mathscr{K}_{\varepsilon}$ открыто и плотно $C^{*}\left(X, \mathbb{R}^{m}\right)$.

Лемма 4.1. Пусть компактное подмножество $A \subset X$ имеет котип $\left(q, \varepsilon, g_{0}\right)$ для некоторьх $g_{0} \in C^{*}\left(X, \mathbb{R}^{m}\right)$ u $\varepsilon>0$. Тогда существуют окрестность $U$ компакта $A$ в $X$ и $\delta>0$ mакие, что $U$ имеет котип $(q, \varepsilon, g)$ для всякого отображения $g \in C^{*}\left(X, \mathbb{R}^{m}\right)$ такого, что $g \mid U \quad \delta$-близко к $g_{0} \mid U$.

ДокАЗАтЕЛьство. Предположим, что лемма неверна. Для всякого $i \geqslant 1$ выберем такую $1 / i$-окрестность $U_{i}$ компакта $A$, что $g_{0}\left(U_{i}\right)$ лежит внутри $1 / i$-окрестности множества $g_{0}(A)$ в $\mathbb{R}^{m}$. Тогда существуют $g_{i} \in C^{*}\left(X, \mathbb{R}^{m}\right)$ и $d$-плоскость $\Pi_{i}^{d} \in \mathscr{P}$ такие, что $g_{i} \mid U_{i} 1 / i$-близко к $g_{0} \mid U_{i}$, но $g_{i}^{-1}\left(\Pi_{i}^{d}\right) \cap U_{i}$ не покрывается никаким семейством $\leqslant q$ открытых дизъюнктных множеств в $X$ диаметра $\leqslant \varepsilon$. Как в доказательстве леммы 3.1, переходя к подпоследовательностям, мы можем предполагать, что $\left\{\Pi_{i}^{d}\right\}_{i=1}^{\infty}$ сходится к некоторой $d$-плоскости $\Pi_{0}^{d}$. Так как все $\Pi_{i}^{d}$ лежат в $\mathscr{P}$ (т.е. параллельны $\Pi^{t} \subset \Pi^{T}$ ), то $\Pi_{0}^{d} \in \mathscr{P}$. Следовательно, множество $A \cap g_{0}^{-1}\left(\Pi_{0}^{d}\right)$ покрывается семейством $\left\{V_{j}\right\}$ мощности $\leqslant q$ открытых попарно дизъюнктных подмножеств $X$ диаметра $\leqslant \varepsilon$. Выберем такие точки $x_{i} \in g_{i}^{-1}\left(\Pi_{i}^{d}\right) \cap$ $\left(U_{i} \backslash V\right)$ и $y_{i} \in A$, что $\operatorname{dist}\left(x_{i}, y_{i}\right) \leqslant 1 / i, i=1,2, \ldots$, где $V=\bigcup V_{j}$. Мы можем 
предполагать, что последовательность $x_{i}$ сходится к некоторой точке $x_{0} \in A$ (напомним, что $A$ компактно). Тогда $\left\{g_{i}\left(x_{i}\right)\right\}$ сходится к $g_{0}\left(x_{0}\right)$ и $g_{0}\left(x_{0}\right) \in g_{0}(A) \cap \Pi_{0}^{d}$. Следовательно, $x_{0} \in A \cap g_{0}^{-1}\left(\Pi_{0}^{d}\right) \subset V$. Таким образом, $x_{i} \in V$ для почти всех $i$. Получили противоречие.

Доказательство следующего следствия похоже на доказательство следствия 3.2 .

СЛЕДСТВИЕ 4.2. Пусть для точки $y_{0} \in Y$ и отображсения $g_{0} \in C^{*}\left(X, \mathbb{R}^{m}\right)$ множество $f^{-1}\left(y_{0}\right)$ имеет котип $\left(q, \varepsilon, g_{0}\right)$. Тогда существуют окрестность $V$ точки у в в $Y$ и $\delta>0$ такие, что множество $f^{-1}(V)$ имеет котип $(q, \varepsilon, g)$ для всякого такого отображсения $g \in C^{*}\left(X, \mathbb{R}^{m}\right)$, ито $g \mid f^{-1}(V)$ и $g_{0} \mid f^{-1}(V)$

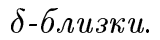

ПРЕДЛОЖЕНИЕ 4.3. Всякое множсество $\mathscr{K}_{\varepsilon}$ открыто в $C^{*}\left(X, \mathbb{R}^{m}\right)$.

Доказательство следует рассуждениям доказательства предложения 3.3, но теперь вместо следствия 3.2 мы применяем следствие 4.2 .

ЛЕмма 4.4. Пусть $M$ - метризуемый не более чем $n$-мерный компакт. Тогда множество $\mathscr{K}_{0}\left(M, \mathbb{R}^{m}\right)$ всех таких отображсений $g \in C\left(M, \mathbb{R}^{m}\right)$, что множество $g^{-1}\left(\Pi^{d}\right)$ состоит из не более чем q точек для всякой плоскости $\Pi^{d} \in \mathscr{P}$, плотно в $C\left(M, \mathbb{R}^{m}\right)$.

ДокаЗАТЕЛЬство. Пусть $\Omega$ - семейство всех таких наборов $\left\{\bar{V}_{1}, \bar{V}_{2}, \ldots, \bar{V}_{q+1}\right\}$ из $q+1$ попарно дизъюнктных элементов, что $V_{j}$ принадлежит фиксированной счетной базе $M$. Пусть также для $\Gamma \in \Omega C_{\Gamma}=\left\{g \in C\left(M, \mathbb{R}^{m}\right): g^{-1}\left(\Pi^{d}\right)\right.$ пересекает не более $q$ элементов $\Gamma$ для всякой плоскости $\left.\Pi^{d} \in \mathscr{P}\right\}$.

Очевидно, что $\Omega$ счетно и $\mathscr{K}_{0}\left(M, \mathbb{R}^{m}\right)$ является пересечением всех множеств $C_{\Gamma}$, $\Gamma \in \Omega$. Следовательно, наше доказательство редуцировано к проверке, что всякое $C_{\Gamma}$ плотно и открыто в $C\left(M, \mathbb{R}^{m}\right)$.

УТВЕРЖДЕНИЕ 1. Всякое множсество $C_{\Gamma}$ открыто в $C\left(M, \mathbb{R}^{m}\right)$.

ДокаЗАтЕЛьство. Фиксируем $\Gamma \in \Omega$ и $g_{0} \in C_{\Gamma}$. Предположим, что для всякого $i$ существует $g_{i} \notin C_{\Gamma}$ такое, что $g_{i} 1 / i$-близко к $g_{0}$. Таким образом, мы можем найти такую плоскость $\Pi_{i}^{d} \in \mathscr{P}$, что множество $g_{i}^{-1}\left(\Pi_{i}^{d}\right)$ пересекает всякий элемент Г. Как в лемме 3.1, мы можем предполагать, что последовательность $\left\{\Pi_{i}^{d}\right\}$ сходится к некоторой плоскости $\Pi_{0}^{d} \in \mathscr{P}$. Тогда $g_{0}^{-1}\left(\Pi_{0}^{d}\right)$ пересекает не более $q$ элементов $\Gamma$, например первые $q$. Теперь для всякого $i$ выберем точку $x_{i} \in g_{i}^{-1}\left(\Pi_{i}^{d}\right) \cap \bar{V}_{q+1}$ и, так как $M$ компактно, мы можем предполагать, что последовательность $\left\{x_{i}\right\}$ сходится к некоторой точке $x_{0} \in \bar{V}_{q+1}$. Тогда $\left\{g_{i}\left(x_{i}\right)\right\}$ сходится к $g_{0}\left(x_{0}\right) \in \Pi_{0}^{d}$. Таким образом, $x_{0} \in g_{0}^{-1}\left(\Pi_{0}^{d}\right) \cap \bar{V}_{q+1}$. Получили противоречие.

УТВЕРЖДЕНИЕ 2. Всякое множество $C_{\Gamma}$ плотно в $C\left(M, \mathbb{R}^{m}\right)$.

ДокаЗАТЕЛЬство. Пусть $\Gamma=\left\{\bar{V}_{1}, \bar{V}_{2}, \ldots, \bar{V}_{q+1}\right\}, g_{0} \in C_{\Gamma}$ и $\delta>0$. Тогда существуют открытое покрытие $\omega$ компакта $M$ такое, что $\operatorname{mesh}(\omega) \leqslant r / 3$, где $r=\min \left\{\operatorname{dist}\left(\bar{V}_{i}, \bar{V}_{j}\right): i \neq j\right\}$, и кусочно линейное отображение $h: L \rightarrow \mathbb{R}^{m}$ такое, что $g=h \circ \pi$ и $g_{0} \delta$-близки. Здесь $L$ - полиэдр нерва $\omega$ и $\pi: M \rightarrow L$-каноническое отображение. Согласно следствию 1.2 мы можем предполагать, что для всякой плоскости $\Pi^{d} \in \mathscr{P}$ число попарно дизъюнктных не более чем $n$-мерных симплексов $\sigma \in L$, образы которых $h(\sigma)$ пересекают $\Pi^{d}$, не превосходит $q$. Мы также можем предполагать, что покрытие $\omega$ имеет порядок $\leqslant n+1$, т.е. $L$ не более чем $n$-мерно. 
Если сушествует такая плоскость $\Pi^{*} \in \mathscr{P}$, что множество $g^{-1}\left(\Pi^{*}\right)$ пересекает всякое $\bar{V}_{i}$, то выберем $x_{i} \in g^{-1}\left(\Pi^{*}\right) \cap \bar{V}_{i}$. Пусть $\omega_{i}-$ семейство тех элементов $\omega$, которые содержат точку $x_{i}$. Тогда всякое семейство $\omega_{i}, i=1, \ldots, q+1$, порождает такой симплекс $\sigma_{i} \in L$ размерности $\leqslant n$, что $h\left(x_{i}\right) \in h\left(\sigma_{i}\right) \cap \Pi^{*}$ и $\sigma_{i} \cap \sigma_{j}=\varnothing$ при $i \neq j$. Это противоречит выбору отображения $h$.

Так как $\mathscr{K}_{0}\left(M, \mathbb{R}^{m}\right) \subset \mathscr{K}_{\varepsilon}\left(M, \mathbb{R}^{m}\right)$ для всякого $\varepsilon>0$, где $\mathscr{K}_{\varepsilon}\left(M, \mathbb{R}^{m}\right)$ является множеством всех таких отображений $g \in C^{*}\left(M, \mathbb{R}^{m}\right)$, что $M$ имеет котип $(q, \varepsilon, g)$, то лемма 4.4 влечет следующее

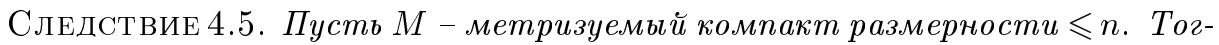
да всякое множество $\mathscr{K}_{\varepsilon}\left(M, \mathbb{R}^{m}\right)$ плотно в $C\left(M, \mathbb{R}^{m}\right)$.

Следующее предложение вместе с предложением 4.3 завершают доказательство теоремы 1.5.

ПРЕДЛОЖЕНИЕ 4.6. Всякое множество $\mathscr{K}_{\varepsilon}$ плотно в $C^{*}\left(X, \mathbb{R}^{m}\right)$.

ДокАЗАТЕЛЬСТво. Пусть $\psi_{\varepsilon}: Y \rightarrow C^{*}\left(X, \mathbb{R}^{m}\right)$ - многозначное отображение $\psi_{\varepsilon}(y)=C^{*}\left(X, \mathbb{R}^{m}\right) \backslash \mathscr{K}_{\varepsilon}(y)$, где $\mathscr{K}_{\varepsilon}(y)$ обозначает множество всех таких $g \in$ $C^{*}\left(X, \mathbb{R}^{m}\right)$, что $f^{-1}(y)$ имеет котип $(q, \varepsilon, g)$. Далее доказательство следует рассуждениям из доказательства предложения 3.5. Для того чтобы показать, что график $\psi_{\varepsilon}$ замкнут, мы воспользуемся следствием 4.2 вместо следствия 3.2. И далее вместо леммы 3.4 необходимо воспользоваться следствием 4.5.

ДОКАЗАТЕЛЬСТво ТЕОРЕМЫ 1.8 . МЫ хотим показать, что $C^{*}\left(X, l_{2}\right)$ содержит плотное $G_{\delta}$-множество таких отображений $g$, что для всякой точки $y \in Y$ и всякой плоскости $\Pi^{d} \in \mathscr{P}(d, r)$ множество $f^{-1}(y) \cap g^{-1}\left(\Pi^{d}\right)$ имеет не более $d+1-r$ точек при $r \leqslant d$ и не более одной точки в противном случае. Для заданных чисел $d, r$ пусть $\mathscr{Q}$ является множеством всех $d$-плоскостей, параллельных $r$-плоскости первых $r$ координат в $l_{2}$. Как в теореме 1.5 , мы вводим понятие подмножества метрического пространства $M$ котипа $(q, \varepsilon, g)$, рассматривая теперь плоскости $\Pi^{d} \in \mathscr{Q}$ и полагая $q=d+1-r$ в случае $d \geqslant r$ и $q=1$ в противном случае. Пусть $\mathscr{F}_{\varepsilon}, \varepsilon>0$, обозначает множество всех таких отображений $g \in C^{*}\left(X, l_{2}\right)$, что $f^{-1}(y)$ имеет котип $(q, \varepsilon, g)$ для всякой точки $y \in Y$. Так как в $l_{2}$ имеется счетное множество координатных $r$-плоскостей, то достаточно показать, что всякое множество $\mathscr{F}_{\varepsilon}$ открыто и плотно в $C^{*}\left(X, l_{2}\right)$. Следуя доказательству теоремы 1.5, можно показать, что все множества $\mathscr{F} \varepsilon$ открыты в $C^{*}\left(X, l_{2}\right)$. Для доказательства плотности $\mathscr{F} \varepsilon$ мы воспользуемся следующей леммой.

Лемма 4.7. Пусть $M-$ метризуемый компакт $и \varepsilon>0$. Тогда множество $\mathscr{F}_{\varepsilon}\left(M, l_{2}\right)$ всех таких отображений $g \in C\left(M, l_{2}\right)$, что $M$ имеет котип $(q, \varepsilon, g)$, плотно в $C\left(M, l_{2}\right)$.

Доказательство. Возьмем $g_{0} \in C\left(M, l_{2}\right)$ и $\lambda>0$. Тогда сушествуют конечный комплекс $K$ и такие отображения $\varphi: M \rightarrow K$ и $h: K \rightarrow l_{2}$, что $h \circ \varphi$ $\lambda / 2$-близко к $g_{0}$. Более того, мы можем предполагать, что все слои отображения $\varphi$ имеют диаметр $<\varepsilon$. Для всякого $A \subset \mathbb{N}$ мы отождествляем $\mathbb{R}^{A}$ с подпространством $\left\{y \in l_{2}: y_{i}=0\right.$ для всех $\left.i \notin A\right\}$ гильбертова пространства $l_{2}$ и обозначаем через $\pi_{A}$ каноническую проекцию $\pi_{A}: l_{2} \rightarrow \mathbb{R}^{A}$. Пусть $\operatorname{dim} K=n$ и $A$ - некоторое конечное подмножество $A \subset \mathbb{N}$, удовлетворяющее следующим условиям:

(i) $\{1, \ldots, r\} \subset A$;

(ii) $|A| \geqslant r+d+2 n+n \cdot|d-r|+1$, где $|A|$ обозначает мошность $A$. 
Так как всякая проекция $\pi_{A}$ является открытьм отображением, то можно показать, что отображение $\Lambda_{A}: C\left(K, l_{2}\right) \rightarrow C\left(K, \mathbb{R}^{A}\right), \Lambda_{A}(g)=\pi_{A} \circ g$, также является открытым.

Предположим сначала, что $r \leqslant d$. Заметим, что если $T=m$, то $(m-n-T) \times$ $(d-r)=-n(d-r) \leqslant 0$, поэтому $n \geqslant(m-n-T)(d-r)$. Следовательно, применяя теорему $1.5 \mathrm{c} m=T=|A|$ и $t=r$, найдем плотное $G_{\delta}$-подмножество $\mathscr{F}_{A}$ в $C\left(K, \mathbb{R}^{A}\right)$, состоящее из таких отображений $g$, что множество $g^{-1}\left(\Pi_{A}^{d}\right)$ содержит не более $1+d-r+\frac{n+n(d-r)}{|A|-n-d}$ точек для всякой $d$-плоскости $\Pi_{A}^{d} \subset \mathbb{R}^{A}$, параллельной $r$-плоскости первых $r$ координат в $\mathbb{R}^{A}$. Так как $|A| \geqslant r+d+2 n+n \cdot|d-r|+1$, то прообраз всякой такой плоскости содержит не более $1+d-r$ точек. Множество $\Lambda_{A}^{-1}\left(\mathscr{F}_{A}\right)$ также плотно и имеет тип $G_{\delta}$ в $C\left(K, l_{2}\right)$. Следовательно, существует отображение $g \in \Lambda_{A}^{-1}\left(\mathscr{F}_{A}\right)$, которое $\lambda / 2$-близко к $h$. Тогда $\bar{g}=g \circ \varphi \quad \lambda$-близко к $g_{0}$. Осталось только показать, что $\bar{g} \in \mathscr{F}_{\varepsilon}\left(M, l_{2}\right)$. Для этого возьмем $\Pi^{d} \in \mathscr{Q}$. Так как $\Pi_{A}^{d}=\pi_{A}\left(\Pi^{d}\right)$ является $d$-плоскостью в $\mathbb{R}^{A}$, параллельной $r$-плоскости первых $r$ координат в $\mathbb{R}^{A}$, то множество $g^{-1}\left(\pi_{A}^{-1}\left(\Pi_{A}^{d}\right)\right)$ содержит $\leqslant 1+d-r$ точек. Формально плоскость $\Pi_{A}^{d}$ может иметь размерность $<d$, но в этом случае оценка на число точек в прообразе только улучшается. Из включения $\Pi^{d} \subset \pi_{A}^{-1}\left(\Pi_{A}^{d}\right)$ следует, что и множество $g^{-1}\left(\Pi^{d}\right)$ содержит $\leqslant 1+d-r$ точек. Поэтому множество $(\bar{g})^{-1}\left(\Pi^{d}\right)=\varphi^{-1}\left(g^{-1}\left(\Pi^{d}\right)\right)$ состоит из $\leqslant 1+d-r$ слоев отображения $\varphi$. Так как всякий слой отображения $\varphi$ имеет диаметр $<\varepsilon$, то $\bar{g} \in \mathscr{F}_{\varepsilon}\left(M, l_{2}\right)$.

Предположим, что $d \leqslant r$. Опять применяем теорему $1.5 \mathrm{k} A$, но теперь при $m=|A|, t=0$ и $T=r$. Очевидно, что в этом случае $(m-n-T)(d-t)>n$. Следовательно, существует плотное $G_{\delta}$-подмножество $\mathscr{F}_{A}$ в $C\left(K, \mathbb{R}^{A}\right)$, состоящее из таких отображений $g$, что $g^{-1}\left(\Pi_{A}^{d}\right)$ содержит не более одной точки для всякой $d$-плоскости в $\mathbb{R}^{A}$, параллельной плоскости первых $r$ координат в $\mathbb{R}^{A}$. Как ранее, берем отображение $g \in \Lambda_{A}^{-1}\left(\mathscr{F}_{A}\right)$, которое $\lambda / 2$-близко к $h$, и показываем, что $\bar{g}=g \circ \varphi \in \mathscr{F}_{\varepsilon}\left(M, l_{2}\right)$.

ПРЕДЛОЖЕНИЕ 4.8. Всякое множество $\mathscr{F} \varepsilon$ плотно в $C^{*}\left(X, l_{2}\right)$.

ДокАЗАТЕЛЬСтво. Мы будем следовать доказательству предложения 3.5, но опишем все необходимые изменения. В нашей ситуации $\psi_{\varepsilon}: Y \rightarrow C^{*}\left(X, l_{2}\right)$ определяется формулой $\psi_{\varepsilon}(y)=C^{*}\left(X, l_{2}\right) \backslash \mathscr{F}_{\varepsilon}(y)$, где $\mathscr{F}_{\varepsilon}(y)$ обозначает множество всех таких отображений $g \in C^{*}\left(X, l_{2}\right)$, что $f^{-1}(y)$ имеет котип $(q, \varepsilon, g)$ с $q=1+d-r$ при $r \leqslant d$ и с $q=1$ в противном случае. Чтобы показать, что $\psi_{\varepsilon}$ имеет замкнутый график, мы вместо следствия 3.2 применим аналог следствия 4.2 к пространству $C^{*}\left(X, l_{2}\right)$. Нам понадобится также следующее

УтВеРЖДЕНИЕ. Пусть $g_{0} \in C^{*}\left(X, l_{2}\right), \alpha: X \rightarrow(0, \infty) u y \in Y$. Тогда для всякого $\varepsilon>0 \quad \psi_{\varepsilon}(y) \cap \bar{B}\left(g_{0}, \alpha\right)$ является $Z$-множеством в $\bar{B}\left(g_{0}, \alpha\right)$, если $\bar{B}\left(g_{0}, \alpha\right)$ рассматривается как подмножсество $C^{*}\left(X, l_{2}\right)$ в топологии равномерной сходимости.

ДоКАЗАТЕЛЬСТВО этого утверждения частично следует рассуждениям из доказательства [13; лемма 8]. Нам необходимо показать, что всякое отображение $h: Q \rightarrow \bar{B}\left(g_{0}, \alpha\right)$, где $Q$ - гильбертов куб, можно аппроксимировать отображением $h_{1}: Q \rightarrow \bar{B}\left(g_{0}, \alpha\right)$, избегающим множество $\psi_{\varepsilon}(y) \cap \bar{B}\left(g_{0}, \alpha\right)$. Итак, фиксируем такое отображение $h$ и $\eta>0$. Тогда $h$ порождает такое отображение $u: Q \times X \rightarrow l_{2}$, $u(z, x)=h(z)(x)$, что $\operatorname{dist}\left(u(z, x), g_{0}(x)\right) \leqslant \alpha(x)$ для всякой точки $(z, x) \in Q \times X$. Выберем такое $\lambda \in(0,1)$, что $\lambda \sup \left\{\alpha(x): x \in f^{-1}(y)\right\}<\eta / 2$, и определим 
$u_{1} \in C\left(Q \times f^{-1}(y), l_{2}\right)$ формулой $u_{1}(z, x)=(1-\lambda) u(z, x)+\lambda g_{0}(x)$. Для всякой точки $(z, x) \in Q \times f^{-1}(y)$ мы имеем

$$
\operatorname{dist}\left(u_{1}(z, x), g_{0}(x)\right)<\alpha(x) \quad \text { и } \quad \operatorname{dist}\left(u_{1}(z, x), u(z, x)\right)<\frac{\eta}{2} .
$$

Пусть $\delta<\inf \left\{\eta / 2, \alpha(x)-\operatorname{dist}\left(u_{1}(z, x), g_{0}(x)\right):(z, x) \in Q \times f^{-1}(y)\right\}$. Согласно лемме 4.7 сушествует такое отображение $u_{2}: Q \times f^{-1}(y) \rightarrow l_{2}$, что $Q \times f^{-1}(y)$ имеет котип $\left(q, \varepsilon, u_{2}\right)$ и $\operatorname{dist}\left(u_{1}(z, x), u_{2}(z, x)\right)<\delta$ для всех $(z, x) \in Q \times f^{-1}(y)$. Тогда

$$
\operatorname{dist}\left(u_{2}(z, x), g_{0}(x)\right)<\alpha(x) \quad \text { и } \quad \operatorname{dist}\left(u_{2}(z, x), u(z, x)\right)<\eta
$$

при условии, что $(z, x) \in Q \times f^{-1}(y)$. Равенство $h_{2}(z)(x)=u_{2}(z, x)$ определяет такое отображение $h_{2}: Q \rightarrow C\left(f^{-1}(y), l_{2}\right)$, что $f^{-1}(y)$ имеет котип $\left(q, \varepsilon, h_{2}(z)\right)$ для всех $z \in Q$. Можно показать, что проектирование $\mathrm{pr}: \bar{B}\left(g_{0}, \alpha\right) \rightarrow C\left(f^{-1}(y), l_{2}\right)$, $\operatorname{pr}(g)=g \mid f^{-1}(y)$, открыто по отношению к топологии равномерной сходимости и $\operatorname{pr}\left(\bar{B}\left(g_{0}, \alpha\right)\right)$ содержит $h_{2}(Q)$. Отсюда следует, что $h_{2}$ может быть поднято до такого отображения $w: Q \rightarrow \bar{B}\left(g_{0}, \alpha\right)$, что $w \eta$-близко к $h$. Заметим, что $w$ избегает множество $\psi_{\varepsilon}(y) \cap \bar{B}\left(g_{0}, \alpha\right)$, так как $f^{-1}(y)$ имеет котип $\left(q, \varepsilon, h_{2}(z)\right)$ для всех $z \in Q$. Это завершает доказательство утверждения.

Вернемся к доказательству предложения 4.8. Для фиксированного отображения $g \in C^{*}\left(X, l_{2}\right)$ и функции $\alpha: X \rightarrow(0,1)$ рассмотрим многозначное отображение $\phi: Y \rightarrow C^{*}\left(X, l_{2}\right), \phi(y)=\bar{B}(g, \alpha)$, где $C^{*}\left(X, l_{2}\right)$ берется в топологии равномерной сходимости. В соответствии с утверждением выше $\phi(y) \cap \psi_{\varepsilon}(y)$ является $Z$-множеством в $\phi(y)$ для всех $y \in Y$. Кроме того, $Y$ является $C$-пространством, поэтому мы можем применить [14; теорема 1.1$]$ и найти такое отображение $\theta: Y \rightarrow$ $C^{*}\left(X, l_{2}\right)$, что $\theta(y) \in \bar{B}(g, \alpha) \backslash \psi_{\varepsilon}(y)$ для всех $y \in Y$. Наконец, определим отображение $\bar{g} \in C^{*}\left(X, l_{2}\right)$ по формуле $\bar{g}(x)=\theta(f(x)), x \in X$. Тогда $\bar{g} \in \bar{B}(g, \alpha) \cap \mathscr{F}_{\varepsilon}(y)$ для всех $y \in Y$. Следовательно, $\bar{g} \in \bar{B}(g, \alpha) \cap \mathscr{F}_{\varepsilon}$, что и завершает доказательство предложения 4.8 .

Следовательно, доказательство теоремы 1.8 завершено.

\section{§5. Приложение}

Дадим некоторые новые приложения наших результатов.

ТЕОРема 5.1. Пусть $f: X \rightarrow Y$ - совершенное отображение паракомпактных пространств $и \operatorname{dim} Y=0$. Пусть $\left\{F_{i}\right\}-$ последовательность замкнутых подмножеств $X u\left\{n_{i}\right\}$ - такая последовательность чельх чисел, что $\operatorname{dim} f \mid F_{i} \leqslant n_{i}$ для всех $i$. Если $n \geqslant n_{i}, i=1,2, \ldots$, то для всякого $m \geqslant n+1$ пространство $C^{*}\left(X, \mathbb{R}^{m}\right)$ содержит плотное $G_{\delta}$-подмножество таких отображсений $g$, что для всякого $i$ и всякой $d$-мерной плоскости $\Pi^{d} \subset \mathbb{R}^{m}$, где $m-n_{i} \leqslant d \leqslant m$, для всякой точки $y \in Y$ справедливо неравенство

$$
\operatorname{dim} g\left(f^{-1}(y) \cap F_{i}\right) \cap \Pi^{d} \leqslant n_{i}+d-m .
$$

ДОКАЗАТЕЛЬСТво ТЕОРЕмЫ 5.1 основано на нижеследующей лемме 5.2. Действительно, для всякого $i$ мы применяем теорему 1.3 к пространствам $F_{i}, f\left(F_{i}\right)$ и отображению $f \mid F_{i}$, чтобы заключить, что $C^{*}\left(F_{i}, \mathbb{R}^{m}\right)$ содержит плотное $G_{\delta^{-}}$множество $\mathscr{H}_{i}$ таких отображений $g$, что $\operatorname{dim} g\left(f^{-1}(y) \cap F_{i}\right) \cap \Pi^{d} \leqslant n_{i}+d-m$ 
для всякой точки $y \in f\left(F_{i}\right)$ и всякой $d$-плоскости $\Pi^{d} \subset \mathbb{R}^{m}$ с $m-n_{i} \leqslant d \leqslant m$. Следовательно, так как согласно лемме 5.2 всякое отображение ограничения

$$
\pi_{i}: C^{*}\left(X, \mathbb{R}^{m}\right) \rightarrow C^{*}\left(F_{i}, \mathbb{R}^{m}\right)
$$

открыто, то множество $\mathscr{K}_{i}=\pi_{i}^{-1}\left(\mathscr{H}_{i}\right)$ плотно и имеет тип $G_{\delta}$ в $C^{*}\left(X, \mathbb{R}^{m}\right)$. Тогда пересечение всех $\mathscr{K}_{i}$ удовлетворяет требованиям теоремы 5.1.

ЛЕмма 5.2. Пусть $F$ - замкнутое подмножсество нормального пространства $X$ и $m \geqslant 1$. Тогда отображсение ограничения $\pi: C^{*}\left(X, \mathbb{R}^{m}\right) \rightarrow C^{*}\left(F, \mathbb{R}^{m}\right)$, определенное формулой $\pi(g)=g \mid F$, открыто, есликак $C^{*}\left(X, \mathbb{R}^{m}\right)$, так и $C^{*}\left(F, \mathbb{R}^{m}\right)$ рассматриваются одновременно в основной предельной топологии или топологии равномерной сходимости.

Нижеприведенное следствие теоремы 5.1 может быть установлено тем же путем, каким следствие 1.4 было получено из теоремы 1.3.

СЛЕДСТВИЕ 5.3. Пусть $\left\{F_{i}\right\}$ - последовательность замкнутых подмножеств нормального пространства $X$ c $\operatorname{dim} F_{i} \leqslant n_{i}$. Ecли $m \geqslant n_{i}+1$ для всех $i$, то $C^{*}\left(X, \mathbb{R}^{m}\right)$ в топологии равномерной сходимости содерәит плотное $G_{\delta}$-подмножество таких отображений $g$, что множество $\overline{g\left(F_{i}\right)} \cap$ $\Pi^{d}$ не более чем $\left(n_{i}+d-m\right)$-мерно для всякой $d$-плоскости $\Pi^{d} \subset \mathbb{R}^{m}$ $c m-n_{i} \leqslant d \leqslant m, i=1,2, \ldots$.

Наше последнее приложение является аналогом теоремы Фокса [15] об экономном продолжении отображений. Если $A$ - замкнутое подмножество пространства $X$ и $h \in C^{*}\left(A, \mathbb{R}^{m}\right)$, то $C_{h}^{*}\left(X, \mathbb{R}^{m}\right)$ обозначает множество всех таких отображений $g \in C^{*}\left(X, \mathbb{R}^{m}\right)$, что $g \mid A=h$. Всюду ниже $C_{h}^{*}\left(X, \mathbb{R}^{m}\right)$ рассматривается как подпространство $C^{*}\left(X, \mathbb{R}^{m}\right)$ в топологии равномерной сходимости.

СЛЕДСТВИЕ 5.4. Пусть $X$ - нормальное пространство и $A$-замкнутое $G_{\delta}$-подмножество $X c \operatorname{dim}(X \backslash A) \leqslant n$. Тогда для всякого $m \geqslant n+1 u$ $h \in C^{*}\left(A, \mathbb{R}^{m}\right)$ существует плотное $G_{\delta}$-подмнодество $C_{h}^{*}\left(X, \mathbb{R}^{m}\right)$ таких отображений $g$, что множество $g(X \backslash A) \cap \Pi^{d}$ не более чем $(n+d-m)$-мерно для всякой $d$-мерной плоскости $\Pi^{d} \subset \mathbb{R}^{m}$ с $m-n \leqslant d \leqslant m$.

ДокАЗАТЕЛЬство. Пусть $\left\{F_{i}\right\}$ - такая последовательность замкнутых подмножеств $X$, что $X \backslash A=\bigcup_{i=1}^{\infty} F_{i}$ и $h \in C^{*}\left(A, \mathbb{R}^{m}\right)$. Согласно следствию 1.4 всякое $C^{*}\left(F_{i}, \mathbb{R}^{m}\right)$ содержит плотное $G_{\delta}$-подмножество $\mathscr{H}_{i}$ таких отображений $g$, что

$$
\operatorname{dim} \overline{g\left(F_{i}\right)} \cap \Pi^{d} \leqslant n+d-m
$$

для всякой $d$-плоскости $\Pi^{d} \subset \mathbb{R}^{m}$ при $m-n \leqslant d \leqslant m$.

Пользуясь тем, что $F_{i}$ и $A$ - это замкнутые дизъюнктные подмножества $X$, можно показать, что всякая проекция $p_{i}: C_{h}^{*}\left(X, \mathbb{R}^{m}\right) \rightarrow C^{*}\left(F_{i}, \mathbb{R}^{m}\right), p_{i}(g)=g \mid F_{i}$, является открытым сюръективным отображением, когда оба пространства $C_{h}^{*}\left(X, \mathbb{R}^{m}\right)$ и $C^{*}\left(F_{i}, \mathbb{R}^{m}\right)$ наделены топологией равномерной сходимости. Следовательно, множество $\mathscr{K}_{i}=p_{i}^{-1}\left(\mathscr{H}_{i}\right)$ плотно и имеет тип $G_{\delta}$ в $C_{h}^{*}\left(X, \mathbb{R}^{m}\right)$. Так как $C_{h}^{*}\left(X, \mathbb{R}^{m}\right)$ обладает свойством Бэра, то $\mathscr{K}=\bigcap_{i=1}^{\infty} \mathscr{K}_{i}$ является плотным $G_{\delta}$-подмножеством в $C_{h}^{*}\left(X, \mathbb{R}^{m}\right)$. Теперь заметим, что условие $(9)$ влечет неравенство $\operatorname{dim} g(X \backslash A) \cap$ $\Pi^{d} \leqslant n+d-m$ для всякого отображения $g \in \mathscr{K}$ и всякой $d$-плоскости $\Pi^{d} \subset \mathbb{R}^{m}$ при $m-n \leqslant d \leqslant m$.

В заключение мы дадим некоторые гипотезы и замечания. 
ГИПОТЕЗА 1. Для отображсения конечномерных метризуемых компактов $f: X \rightarrow Y$ пространство $C\left(X, \mathbb{R}^{m}\right)$ содержит плотное $G_{\delta}$-подмножество таких отображений $\varphi$, что для всяких чельх чисел $d, t, T$, $0 \leqslant t \leqslant d \leqslant$ $T \leqslant m u \operatorname{dim} f+d+1 \leqslant m$ и для всякой $d$-мерной плоскости $\Pi^{d} \subset \mathbb{R}^{m}$, параллельной некоторым координатным плоскостям $\Pi^{t} \subset \Pi^{T}$ в $\mathbb{R}^{m}$, всякое множество $f^{-1}(y) \cap \varphi^{-1}\left(\Pi^{d}\right), y \in Y$, имеет не более чем

$$
1+\frac{\operatorname{dim} Y+\operatorname{dim} f+(T-d)(d-t)}{m-\operatorname{dim} f-d}
$$

точек.

Отметим, что теорема 1.5 влечет справедливость гипотезы 1 в случае 0-мерного пространства $Y$.

ГИПОтеЗА 2. Для отображсения конечномерных компактов $f: X \rightarrow Y$ пространство $C\left(X, \mathbb{R}^{m}\right)$ содержит плотное $G_{\delta}$-подмнохество таких отображений $\varphi$, что

$$
\operatorname{dim}\left(\varphi\left(f^{-1}(y)\right) \cap \Pi^{d}\right) \leqslant \operatorname{dim} f+d-m
$$

для всякой $d$-плоскости $\Pi^{d} \subset \mathbb{R}^{m}$ с $m-\operatorname{dim} f \leqslant d \leqslant m$ и всякой точки $у \in Y$.

Теорема 1.3 влечет справедливость гипотезы 2 в случае 0-мерного пространства $Y$. Как и в случае теоремы 1.3 , достаточно установить гипотезу 2 в специальном случае $d=m-\operatorname{dim} f$. Гипотеза 2 справедлива при $\operatorname{dim} f=0$ - это теорема Успенского [16] о легких отображениях. Случай совершенного отображения $f$ паракомпактных пространств с $\operatorname{dim} f>0$ рассматривается в теореме Тункали-Вылова в работе [17].

Пусть $f: X \rightarrow Y, \varphi \in C\left(X, \mathbb{R}^{m}\right)$ и $t, d, T$ - такие целые числа, что $0 \leqslant t \leqslant d \leqslant$ $T \leqslant m$ и $d-t+1 \leqslant q$. Ниже мы рассматриваем множество $B_{q, d, t, T}^{f}(\varphi)$, состоящее из всех точек $\left(y, y_{1}, \ldots, y_{q}\right) \in Y \times\left(\mathbb{R}^{m}\right)^{q}$, удовлетворяющих следующему условию: сушествуют такие точки $x_{1}, \ldots, x_{q} \in f^{-1}(y)$ с $x_{i} \neq x_{j}$ для $i \neq j$, что $y_{i}=\varphi\left(x_{i}\right)$ и все точки $y_{i}, i=1, \ldots, q$, принадлежат некоторой $d$-плоскости в $\mathbb{R}^{m}$, параллельной некоторьм координатньм плоскостям $\Pi^{t} \subset \Pi^{T} \subset \mathbb{R}^{m}$.

ГИПОТЕЗА 3. Для отображения конечномерных метризуемых компактов $f: X \rightarrow Y$ пространство $C\left(X, \mathbb{R}^{m}\right)$ содержит плотное $G_{\delta}$-подмножество $\mathscr{H}$ таких отображений $\varphi$, что

$$
\operatorname{dim} B_{q, d, t, T}^{f}(\varphi) \leqslant \operatorname{dim} Y+\operatorname{dim} f+(T-d)(d-t)-(q-1)(m-\operatorname{dim} f-d)
$$

для всех иельх чисел $d, t, T, q$, удовлетворяющих условиям $0 \leqslant t \leqslant d \leqslant T \leqslant m$, $\operatorname{dim} f+d+1 \leqslant m u d-t+1 \leqslant q$.

Уточним, что если в гипотезе 3 правая часть неравенства $\leqslant-1$, то это означает, что множество $B_{q, d, t, T}^{f}(\varphi)$ пусто. Именно условия, при которых множество $B_{q, d, t, T}^{f}(\varphi)$ пусто, обсуждаются в гипотезе 1 . Если $d=0$, то множество $B_{q, 0,0, T}^{f}(\varphi)$ не зависит от $T$. В этом случае оно гомеоморфно множеству $B_{q}^{f}(\varphi)=\{(y, z) \in Y \times$ $\left.\mathbb{R}^{m}:\left|f^{-1}(y) \cap \varphi^{-1}(z)\right| \geqslant q\right\}$. В случае $d=0$ и одноточечного пространства $Y$ гипотеза 3 была получена Гуревичем в 1933 году [18] (напомним, что отображения $\varphi \in \mathscr{H}$ из теоремы Гуревича называются регулярно ветвящимися отображсениями [19]). Параметрическая версия теоремы Гуревича была получена в [20; теорема 1.1]. Согласно терминологии работы [20] неравенство из гипотезы 3 удовлетворяется при $d=0$ для всех $q \geqslant 1$ тогда и только тогда, когда отображение $\varphi$ 
является $f$-регулярно ветвящимся. Так как согласно [20; теорема 1.1] пространство $C\left(X, \mathbb{R}^{m}\right)$ содержит плотное $G_{\delta}$-подмножество $f$-регулярно ветвящихся отображений, то гипотеза 3 справедлива при $d=0$. Подчеркнем, что при $d \geqslant 1$ даже в случае одноточечного пространства $Y$, т.е. непараметрическая версия, гипотеза открыта.

Для отображений $f_{i}: X_{i} \rightarrow \mathbb{R}^{m}, i=1, \ldots, q$, и целых чисел $0 \leqslant t \leqslant d \leqslant T \leqslant m$ пусть $B_{d, t, T}\left(f_{1}, \ldots, f_{q}\right)$ - это множество всех таких точек $\left(y_{1}, \ldots, y_{q}\right) \in\left(\mathbb{R}^{m}\right)^{q}$, что точки $y_{i}=f\left(x_{i}\right), i=1, \ldots, q$, принадлежат некоторой $d$-плоскости в $\mathbb{R}^{m}$, параллельной некоторым координатным плоскостям $\Pi^{t} \subset \Pi^{T}$. Пусть также

$$
\begin{aligned}
C_{d, t, T}\left(f_{1}, \ldots, f_{q}\right)= & \left\{\left(x_{1}, \ldots, x_{q}\right) \in X_{1} \times \cdots \times X_{q}:\left(f\left(x_{1}\right), \ldots, f\left(x_{q}\right)\right)\right. \\
& \left.\in B_{d, t, T}\left(f_{1}, \ldots, f_{q}\right)\right\} .
\end{aligned}
$$

ГИПОТЕЗА 4. Пусть целье числа $n_{1}, \ldots, n_{q}, m, d, t, T$ удовлетворяют неравенствам $0 \leqslant t \leqslant d \leqslant T \leqslant m, 0 \leqslant n_{1}, \ldots, 0 \leqslant n_{q}, n_{1}+1+d \leqslant m, \ldots, n_{q}+1+$ $d \leqslant m, d-t+1 \leqslant q u$

$$
n_{1}+\cdots+n_{q} \geqslant(m-d)(q-1)-(T-d)(d-t) .
$$

Тогда существуют $\varepsilon>0$ и такие отображсения $f_{i}: \Delta^{n_{i}} \rightarrow \mathbb{R}^{m}, i=1, \ldots, q$, что мнохество $B_{d, t, T}\left(g_{1}, \ldots, g_{q}\right)$ непусто для любъх таких отображсений $g_{i}: \Delta^{n_{i}} \rightarrow \mathbb{R}^{m}$, что $g_{i}$ и $f_{i}$ є-близки при всех $i=1, \ldots, q$.

В простейшем случае $\left(q=1, n_{1}=0, n_{2}=m, d=0\right)$ утверждение гипотезы 4 это в точности теорема Александрова о том, что тождественное отображение шара на себя является существенным. При $d=0$ гипотеза 4 верна в обшем случае (см., например, [21; следствие 3], [22; лемма 6.3, с. 65]). В случае $d=q-1, t=0$ и $T=m$ утверждение гипотезы 4 доказал Болтянский [9; лемма 9] (основньм элементом его доказательства является то, что если для некоторого $n$-мерного полиэдра $X$ множество всех $k$-регулярных отображений $X$ в $\mathbb{R}^{m}$ плотно в $C\left(X, \mathbb{R}^{m}\right)$, то справедливо неравенство $m \geqslant n k+n+k)$.

ГИПОТЕЗА 5. Пусть челье числа $n_{1}, \ldots, n_{q}, m, d, t, T$ удовлетворяют неравенствам $0 \leqslant t \leqslant d \leqslant T \leqslant m, 0 \leqslant n_{1}, \ldots, 0 \leqslant n_{q}, n_{1}+1+d \leqslant m, \ldots, n_{q}+$ $1+d \leqslant m, d-t+1 \leqslant q$. Тогда существуют $\varepsilon>0$ и такие отображсения $f_{i}: \Delta^{n_{i}} \rightarrow \mathbb{R}^{m}, i=1, \ldots, q$, чmo

$$
\operatorname{dim} C_{d, t, T}\left(g_{1}, \ldots, g_{q}\right) \geqslant n_{1}+\cdots+n_{q}-(m-d)(q-1)+(T-d)(d-t)
$$

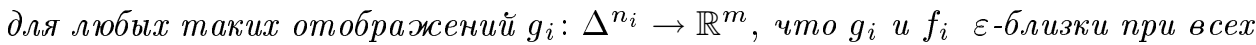
$i=1, \ldots, q$.

Для $d=0$ утверждение гипотезы 5 было установлено в [21; следствие 3$]$.

Завершим все следуюшей проблемой, где $\Delta_{n}^{N}$ обозначает $n$-мерный остов $N$-мерного симплекса $\Delta^{N}$.

ПрОБЛЕМА. Найти все такие челье числа $n, m, q, d, t, T$, что $0 \leqslant n$, $0 \leqslant t \leqslant d \leqslant T \leqslant m, n+1+d \leqslant m, d-t+1 \leqslant q$,

$$
n \geqslant(m-n-d)(q-1)-(T-d)(d-t) \quad(n, m, q, d, t, T)
$$

и выполнено следующее условие: существует такое натуральное $N$, что для всякого отображсния $f: \Delta_{n}^{N} \rightarrow \mathbb{R}^{m}$ имеются попарно дизбюнктные симплексъц $\sigma_{1}, \ldots, \sigma_{q} \subset \Delta_{n}^{N}$, образы которых при $f$ пересекают некоторую $d$-мерную 
плоскость $\Pi^{d} \subset \mathbb{R}^{m}$, параллельную некоторьм координатным плоскостям $\Pi^{t} \subset \Pi^{T}$.

Заметим, что если эта проблема имеет положительное решение для некоторых чисел $n, m, q, d, t, T$, удовлетворяющих предположениям проблемы, то следствие 1.6 нельзя улучшить не только на уровне плотного множества отображений, а даже на уровне существования одного малократного отображения, причем уже в классе полиэдров. Большинство результатов о существовании такого числа $N$ получили для $d=0$ ван Кампен и Флорес [23], [24] ( $q=2$ и $N=2 n+2)$, Саркария [25] ( $q$ простое и $N=q n+2 q-2)$, Воловиков [26] ( $q$ - степень простого числа и $N=q n+2 q-2)$, Богатый [21; следствие 11] $\left(q=n+1\right.$ и $\left.N \leqslant 2 n^{2}+5 n\right)$. Другой результат Богатого [3], [27] дает такое $N$ при $d=q-1, t=0, T=m$ с нечетным $q$. Живайевич получил [28] некоторую теорему о вложениях двудольного графа $K_{6,6}$ в $\mathbb{R}^{3}$, которая влечет положительное решение проблемы для $n=1, m=3, t=0$, $T=3, q=4$ и $N=11$.

В случае $d=q-1, q=2, t=0$ и $T=m$ не сушествует числа $N$, удовлетворяюшего условиям проблемы (см. [9]).

Сформулированной проблеме можно дать более общую постановку: для данного семейства чельх чисел $n, q, d, t, T$ найти наибольиее число $m$, для которого такое число $N$ существует. Тогда возникает задача нахождения наименьшего такого числа $N$. Более сложной является задача описания в этом случае минимальных таких подполиәдров в $\Delta_{n}^{N}$. При $n=1, d=0, q=2, m=2$ такими минимальными полиэдрами являются графы Куратовского - полный граф $K_{5}$ и полньй двудольный граф̆ $K_{3,3}$.

Отметим, что имеется тесная связь проблемы с гипотезами о различных формах теоремы Тверберга [3], [28], [29]. Хорошо известно также, что $k$-регулярные отображения тесно связаны с интерполяционной и аппроксимационной проблемами [30], [31]. В связи с этим было бы важно найти приложения отображсений, описъваемых теоремами 1.5 и 1.8, в интерполячионной и аппроксимационной проблемах.

\section{Список литературы}

1. Федорчук B. В., Чигогидзе A. Ч. Абсолютные ретракты и бесконечномерные многообразия. М.: Наука, 1992.

2. Богатый С. А. Цветная теорема Тверберг // Вестн. МГУ. Сер. 1. Матем., мех. 1999. T. 3. C. $14-19$

3. Богатыи С. A. Гипотеза Борсука, препятствие Рышкова, интерполяция, аппроксимация Чебьшева, трансверсальная теорема Тверберга, задачи // Труды МИАН. 2002. Т. 239. C. $63-82$.

4. Roberts J. A theorem on dimension // Duke Math. J. 1941. V. 8. P. 565-574.

5. Berkowitz H., Roy P. General position and algebraic independence // Geometric Topology. Proceedings of the Geometry Topology Conference, Park City, UT / ed. L. C. Glaser, T. B. Rushing. New York: Springer, 1975. P. 9-15.

6. Goodsell T. Projections of compacta in $\mathbb{R}^{n} / /$ Ph.D. thesis. Provo, UT: Brigham Young Univ., 1997.

7. Goodsell T. Strong general position and Menger curves // Topology Appl. 2002. V. 120. P. $47-55$.

8. Wright D. G. Geometric taming of compacta in $E^{n} / /$ Proc. Amer. Math. Soc. 1982. V. 86. № 4. P. $641-645$.

9. Болтянский В.Г. Отображения компактов в евклидовы пространства // Изв. АН CCCP. Сер. матем. 1959. Т. 23. С. 871-892.

10. Munkers J. Topology. Englewood Cliffs, NY: Prentice Hall, 1975. 
11. Repovš D., Semenov P. Continuous selections of multivalued mappings. Dordrecht: Kluwer, 1998. (Math. Appl. V. 455.)

12. Michael E. Continuous selections avoiding a set // Topology Appl. 1988. V. 28. P. 195-213.

13. Tuncali M., Valov V. On dimensionally restricted maps // Fund. Math. 2002. V. 175. P. $35-52$.

14. Gutev V., Valov $V$. Dense families of selections and finite-dimensional spaces // Set-Valued Anal. 2003. V. 11. P. 373-391.

15. Fox R. Extension of homeomorphisms into Euclidean and Hilbert parallelotopes // Duke Math. J. 1941. V. 8. P. 452-456.

16. Uspenskij $V$. V. A remark on a question of R. Pol concerning light maps // Topology Appl. 2000. V. 103. № 3. P. 291-293.

17. Tuncali M., Valov V. On finite-dimensional maps. II // Topology Appl. 2003. V. 132. P. 81-87.

18. Hurewicz $W$. Über Abbildungen von endlichdimensionalen Räumen auf Teilmengen Cartesischer Räume // Sitzungsber. Preuss. Akad. Wiss. Phys.-Math. Kl. 1933. V. 4. P. 754-768.

19. Dranišnikov A. N., Repov̌s D., Ščepin E. V. On intersections of compacta of complementery dimensions in Euclidean space // Topol. Appl. 1991. V. 38. P. 237-253.

20. Tuncali M., Valov V. On regularly branched maps // Topology Appl. 2004. V. 145. P. 119-145.

21. Богатый C. А. Геометрия отображений в евклидово пространство // УМН. 1998. Т. 53. № 5. C. $27-56$.

22. Boltyanski V., Martini H., Soltan V. Geometric methods and optimization problems. Dordrecht: Kluwer Acad. Publ., 1999.

23. van Kampen E. R. Komplexe in euklidischen Räumen // Abh. Math. Sem. Univ. Hamburg. 1932. V. 9. P. $72-78$.

24. Flores $A$. Über $n$-dimensionale Komplexe, die $\operatorname{im} \mathbb{R}_{2 n+1}$ absolut selbstverschlungen sind // Ergeb. Math. Kolloq. 1935. V. 6. P. 4-7.

25. Sarkaria K. S. A generalized van Kampen-Flores theorem // Proc. Amer. Math. Soc. 1991. V. 111. № 2. P. 559-565.

26. Воловиков А. Ю. К теореме ван Кампена-Флореса // Матем. заметки. 1996. Т. 59. №5. C. $663-670$.

27. Богатый C.A. $k$-регулярные отображения в евклидово пространство и задача Борсука-Болтянского // Матем. сб. 2002. Т. 193. № 1. С. 73-82.

28. Živaljević $R$. T. The Tverberg-Vrećica problem and the combinatorial geometry on vector bundles // Israel J. Math. 1999. V. 111. P. 53-76.

29. Tverberg H., Vrećica S. On generalizations of Radon's theorem and the ham sandwich theorem // European J. Combin. 1993. V. 14. P. 259-264.

30. Болтянский В. Г., Рьиков С. С., Шашкин Ю.А. О $k$-регулярных вложениях и их применении к теории приближения функций // УМН. 1960. Т. 15. №6. С. 125-132.

31. Шашкин Ю. А. Интерполяционные семейства функций и вложения множеств в евклидовы и проективные пространства // Докл. АН СССР. 1967. V. 174. № 5. Р. 1030-1032.

Московский государственньй университет им. М.В. Ломоносова;

Nipissing University, Canada

E-mail: bogatyi@mech.math.msu.su

veskov@nipissingu.ca 